\title{
Homotopy Theory for Digraphs
}

\author{
Alexander Grigor'YAN*, YONG Lin ${ }^{\dagger}$, YURI MURANOV ${ }^{\ddagger}$ AND \\ SHING-TUNG YAU ${ }^{\S}$
}

\begin{abstract}
We introduce a homotopy theory of digraphs (directed graphs) and prove its basic properties, including the relations to the homology theory of digraphs constructed by the authors in previous papers. In particular, we prove the homotopy invariance of homologies of digraphs and the relation between the fundamental group of the digraph and its first homology group.

The category of (undirected) graphs can be identified by a natural way with a full subcategory of digraphs. Thus we obtain also consistent homology and homotopy theories for graphs. Note that the homotopy theory for graphs coincides with the one constructed in [1] and [2].
\end{abstract}

Keywords: homotopy of digraphs, homology of digraphs, fundamental group of digraphs, homotopy groups of digraphs, homology of graphs, homotopy of graphs, graph coloring, algebraic topology for digraphs.

\section{Introduction}

2 Homology theory of digraphs

Received June 27, 2014.

*Partially supported by SFB 701 of German Research Council.

${ }^{\dagger}$ Supported by the Fundamental Research Funds for the Central Universities and the Research Funds of (11XNI004).

${ }^{\ddagger}$ Partially supported by the CONACyT Grants 98697 and 151338, SFB 701 of German Research Council, and the travel grant of the Commission for Developing Countries of the International Mathematical Union.

§Partially supported by the grant "Geometry and Topology of Complex Networks", no. FA-9550-13-1-0097. 
2.3 Regular paths

2.4 Allowed and $\partial$-invariant paths on digraphs

2.5 Cylinders

3 Homotopy theory of digraphs

3.1 The notion of homotopy

3.2 Homotopy preserves homologies

3.3 Retraction

3.4 Cylinder of a map

642

4 Homotopy groups of digraphs

643

4.1 Construction of $\pi_{0}$

644

4.2 $C$-homotopy and $\pi_{1}$

4.3 Local description of $C$-homotopy

4.4 Group structure in $\pi_{1}$

4.5 Relation between $H_{1}$ and $\pi_{1}$

4.6 Higher homotopy groups

663

5 Application to graph coloring

665

6 Homology and homotopy of (undirected) graphs

666

References

673

\section{Introduction}

The homology theory of digraphs has been constructed in a series of the previous papers of the authors (see, for example, [5], [6], [7]). In the present paper we introduce a homotopy theory of digraphs and prove that there 
are natural relations to aforementioned homology theory. In particular, we prove the invariance of the homology theory under homotopy and the relation between the fundamental group and the first homology group, which is similar to the one in the classical algebraic topology. Let emphasize, that the theories of homology and homotopy of digraphs are introduced entirely independent each other, but nevertheless they exhibit a very tight connection similarly to the classical algebraic topology.

The homotopy theory of undirected graphs was constructed by Babson, Barcelo, Kramer, Laubenbacher, Longueville and Weaver in [1] and [2]. We identify in a natural way the category of graphs with a full subcategory of digraphs, which allows us to transfer the homology and homotopy theories to undirected graphs. The homotopy theory of graphs, obtained in this way, coincides with the homotopy theory constructed in [1] and [2]. However, our notion of homology of graphs is new, and the result about homotopy invariance of homologies of graphs is also new. Hence, our results give an answer to the question raised in [1] asking "for a homology theory associated to the A-theory of a graph".

There are other homology theories on graphs that try to mimic the classical singular homology theory. In those theories one uses predefined "small" graphs as basic cells and defines singular chains as formal sums of the maps of the basic cell into the graph (see, for example, [9], [13]). However, simple examples show that the homology groups obtained in this way, depend essentially on the choice of the basic cells.

Our homology theory of digraphs (and graphs) is very different from the "singular" homology theories. We do not use predefined cells but formulate only the desired properties of the cells in terms of the digraph (graph) structure. Namely, each cell is determined by a sequence of vertices that goes along the edges (allowed paths), and the boundary of the cell must also be of this type. This homology theory has very clear algebraic [6] and geometric [7], [5] motivation. It provides effective computational methods for digraph (graph) homology, agrees with the homotopy theory, and provides good connections with homology theories of simplicial and cubical complexes [7] and, in particular, with homology of triangulated manifolds.

Let us briefly describe the structure of the paper and the main results. In Section 2 we give a short survey on homology theory for digraphs following $[5],[7]$.

In Section 3, we introduce the notion of homotopy of digraphs. We prove the homotopy invariance of homology groups (Theorem 3.3) and give a number of examples based on the notion of deformation retraction. 
In Section 4, we define a fundamental group $\pi_{1}$ of digraph. Elements of $\pi_{1}$ are equivalence classes of loops on digraphs, where the equivalence of the loops is defined using a new notion of $C$-homotopy, which is more general than a homotopy. A description of $C$-homotopy in terms of local transformations of loops is given in Theorem 4.13.

We prove the homotopy invariance of $\pi_{1}$ (Theorem 4.22) and the relation $H_{1}=\pi_{1} /\left[\pi_{1}, \pi_{1}\right]$ between the first homology group over $\mathbb{Z}$ and the fundamental group (Theorem 4.23). We define higher homotopy groups by induction using the notion of a loop digraph.

In Section 5 we give a new proof of the classical Sperner lemma, using fundamental groups of digraphs. We hope that our notions of homotopy and homology theories on digraph can find further applications in graph theory, in particular, in graph coloring.

In Section 6 we construct isomorphism between the category of (undirected) graphs and a full subcategory of digraphs, thus transferring the aforementioned results from the category of digraphs to the category of graphs.

\section{Homology theory of digraphs}

In this Section we state the basic notions of homology theory for digraphs in the form that we need in subsequent sections. This is a slight adaptation of a more general theory from [5], [7].

\subsection{The notion of a digraph}

We start with some definitions.

Definition 2.1. A directed graph (digraph) $G=(V, E)$ is a couple of a set $V$, whose elements are called the vertices, and a subset $E \subset\{V \times V \backslash \operatorname{diag}\}$ of ordered pairs of vertices that are called (directed) edges or arrows. The fact that $(v, w) \in E$ is also denoted by $v \rightarrow w$.

In particular, a digraph has no edges $v \rightarrow v$ and, hence, it is a combinatorial digraph in the sense of [11]. We write

$$
v \rightrightarrows w
$$

if either $v=w$ or $v \rightarrow w$. In this paper we consider only finite digraphs, that is, digraphs with a finite set of vertices. 
Definition 2.2. A morphism from a digraph $G=\left(V_{G}, E_{G}\right)$ to a digraph $H=\left(V_{H}, E_{H}\right)$ is a map $f: V_{G} \rightarrow V_{H}$ such that for any edge $v \rightarrow w$ on $G$ we have $f(v) \rightrightarrows f(w)$ on $H$ (that is, either $f(v) \rightarrow f(w)$ or $f(v)=f(w)$ ). We will refer to such morphisms also as digraphs maps (sometimes simply maps) and denote them shortly by $f: G \rightarrow H$.

The set of all digraphs with digraphs maps form a category of digraphs that will be denoted by $\mathcal{D}$.

Definition 2.3. For two digraphs $G=\left(V_{G}, E_{G}\right)$ and $H=\left(V_{H}, E_{H}\right)$ define the Cartesian product $G \boxminus H$ as a digraph with the set of vertices $V_{G} \times V_{H}$ and with the set of edges as follows: for $x, x^{\prime} \in V_{G}$ and $y, y^{\prime} \in V_{H}$, we have $(x, y) \rightarrow\left(x^{\prime}, y^{\prime}\right)$ in $G \boxminus H$ if and only if

$$
\text { either } x^{\prime}=x \text { and } y \rightarrow y^{\prime} \text {, or } x \rightarrow x^{\prime} \text { and } y=y^{\prime} \text {, }
$$

as is shown on the following diagram:

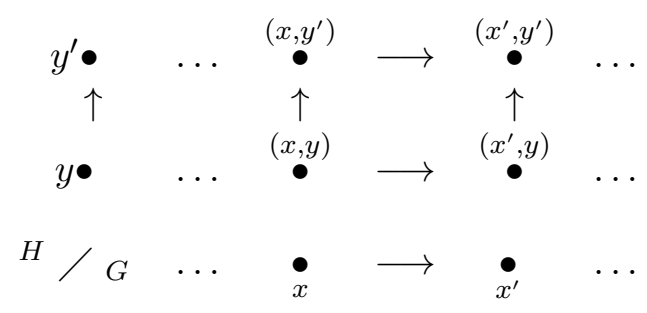

\subsection{Paths and their boundaries}

Let $V$ be a finite set. For any $p \geq 0$, an elementary $p$-path is any (ordered) sequence $i_{0}, \ldots, i_{p}$ of $p+1$ vertices of $V$ that will be denoted simply by $i_{0} \ldots i_{p}$ or by $e_{i_{0} \ldots i_{p}}$. Fix a commutative ring $\mathbb{K}$ with unity and denote by $\Lambda_{p}=$ $\Lambda_{p}(V)=\Lambda_{p}(V, \mathbb{K})$ the free $\mathbb{K}$-module that consist of all formal $\mathbb{K}$-linear combinations of all elementary $p$-paths. Hence, each $p$-path has a form

$$
v=\sum_{i_{0} i_{1} \ldots i_{p}} v^{i_{0} i_{1} \ldots i_{p}} e_{i_{0} i_{1} \ldots i_{p}}, \quad \text { where } v^{i_{0} i_{1} \ldots i_{p}} \in \mathbb{K} .
$$

Definition 2.4. Define for any $p \geq 0$ the boundary operator $\partial: \Lambda_{p+1} \rightarrow \Lambda_{p}$ by

$$
(\partial v)^{i_{0} \ldots i_{p}}=\sum_{k} \sum_{q=0}^{p+1}(-1)^{q} v^{i_{0} \ldots i_{q-1} k i_{q} \ldots i_{p}}
$$


where 1 is the unity of $\mathbb{K}$ and the index $k$ is inserted so that it is preceded by $q$ indices.

Sometimes we need also the operator $\partial: \Lambda_{0} \rightarrow \Lambda_{-1}$ where we set $\Lambda_{-1}=$ $\{0\}$ and $\partial v=0$ for all $v \in \Lambda_{0}$. It follows from (2.1) that

$$
\partial e_{j_{0} \ldots j_{p+1}}=\sum_{q=0}^{p+1}(-1)^{q} e_{j_{0} \ldots \widehat{j_{q} \ldots j_{p+1}}} .
$$

It is easy to show that $\partial^{2} v=0$ for any $v \in \Lambda_{p}([5])$. Hence, the family of $\mathbb{K}$-modules $\left\{\Lambda_{p}\right\}_{p>-1}$ with the boundary operator $\partial$ determine a chain complex that will be denoted by $\Lambda_{*}(V)=\Lambda_{*}(V, \mathbb{K})$.

\subsection{Regular paths}

Definition 2.5. An elementary $p$-path $e_{i_{0} \ldots i_{p}}$ on a set $V$ is called regular if $i_{k} \neq i_{k+1}$ for all $k=0, \ldots, p-1$, and irregular otherwise.

Let $I_{p}$ be the submodule of $\Lambda_{p}$ that is $\mathbb{K}$-spanned by irregular $e_{i_{0} \ldots i_{p}}$. It is easy to verify that $\partial I_{p} \subset I_{p-1}$ (cf. [5]). Consider the quotient $\mathcal{R}_{p}:=\Lambda_{p} / I_{p}$. Since $\partial I_{p} \subset I_{p-1}$, the induced boundary operator

$$
\partial: \mathcal{R}_{p} \rightarrow \mathcal{R}_{p-1} \quad(p \geq 0)
$$

is well-defined. We denote by $\mathcal{R}_{*}(V)$ the obtained chain complex. Clearly, $\mathcal{R}_{p}$ is linearly isomorphic to the space of regular $p$-paths:

$$
\mathcal{R}_{p} \cong \operatorname{span}_{\mathbb{K}}\left\{e_{i_{0} \ldots i_{p}}: i_{0} \ldots i_{p} \text { is regular }\right\}
$$

For simplicity of notation, we will identify $\mathcal{R}_{p}$ with this space, by setting all irregular $p$-paths to be equal to 0 .

Given a map $f: V \rightarrow V^{\prime}$ between two finite sets $V$ and $V^{\prime}$, define for any $p \geq 0$ the induced map

$$
f_{*}: \Lambda_{p}(V) \rightarrow \Lambda_{p}\left(V^{\prime}\right)
$$

by the rule $f_{*}\left(e_{i_{0} \ldots i_{p}}\right)=e_{f\left(i_{0}\right) \ldots f\left(i_{p}\right)}$, extended by $\mathbb{K}$-linearity to all elements of $\Lambda_{p}(V)$. The map $f_{*}$ is a morphism of chain complexes, because it trivially follows from $(2.2)$ that $\partial f_{*}=f_{*} \partial$. Clearly, if $e_{i_{0} \ldots i_{p}}$ is irregular then 
$f_{*}\left(e_{i_{0} \ldots i_{p}}\right)$ is also irregular, so that

$$
f_{*}\left(I_{p}(V)\right) \subset I_{p}\left(V^{\prime}\right)
$$

Therefore, $f_{*}$ is well-defined on the quotient $\Lambda_{p} / I_{p}$ so that we obtain the induced map

$$
f_{*}: \mathcal{R}_{p}(V) \rightarrow \mathcal{R}_{p}\left(V^{\prime}\right)
$$

Since $f_{*}$ still commutes with $\partial$, we see that the induced map (2.4) induces a morphism $\mathcal{R}_{*}(V) \rightarrow \mathcal{R}_{*}\left(V^{\prime}\right)$ of chain complexes. With identification (2.3) of $\mathcal{R}_{p}$ we have the following rule for the map (2.4):

$$
f_{*}\left(e_{i_{0} \ldots i_{p}}\right)= \begin{cases}e_{f\left(i_{0}\right) \ldots f\left(i_{p}\right)}, & \text { if } e_{f\left(i_{0}\right) \ldots f\left(i_{p}\right)} \text { is regular } \\ 0, & \text { if } e_{f\left(i_{0}\right) \ldots f\left(i_{p}\right)} \text { is irregular }\end{cases}
$$

\subsection{Allowed and $\partial$-invariant paths on digraphs}

Definition 2.6. Let $G=(V, E)$ be a digraph. An elementary $p$-path $i_{0} \ldots i_{p}$ on $V$ is called allowed if $i_{k} \rightarrow i_{k+1}$ for any $k=0, \ldots, p-1$, and non-allowed otherwise. The set of all allowed elementary $p$-paths will be denoted by $E_{p}$.

For example, $E_{0}=V$ and $E_{1}=E$. Clearly, all allowed paths are regular. Denote by $\mathcal{A}_{p}=\mathcal{A}_{p}(G)$ the submodule of $\mathcal{R}_{p}(G):=\mathcal{R}_{p}(V)$ spanned by the allowed elementary $p$-paths, that is,

$$
\mathcal{A}_{p}=\operatorname{span}_{\mathbb{K}}\left\{e_{i_{0} \ldots i_{p}}: i_{0} \ldots i_{p} \in E_{p}\right\} .
$$

The elements of $\mathcal{A}_{p}$ are called allowed $p$-paths.

Note that the modules $\mathcal{A}_{p}$ of allowed paths are in general not invariant for $\partial$. Consider the following submodules of $\mathcal{A}_{p}$

$$
\Omega_{p} \equiv \Omega_{p}(G):=\left\{v \in \mathcal{A}_{p}: \partial v \in \mathcal{A}_{p-1}\right\}
$$

that are $\partial$-invariant. Indeed, $v \in \Omega_{p}$ implies $\partial v \in \mathcal{A}_{p-1}$ and $\partial(\partial v)=0 \in$ $\mathcal{A}_{p-2}$, whence $\partial v \in \Omega_{p-1}$. The elements of $\Omega_{p}$ are called $\partial$-invariant $p$-paths.

Hence, we obtain a chain complex $\Omega_{*}=\Omega_{*}(G)=\Omega_{*}(G, \mathbb{K})$ :

$$
0 \leftarrow \Omega_{0} \stackrel{\partial}{\leftarrow} \Omega_{1} \stackrel{\partial}{\leftarrow} \ldots \stackrel{\partial}{\leftarrow} \Omega_{p-1} \stackrel{\partial}{\leftarrow} \Omega_{p} \stackrel{\partial}{\leftarrow} \ldots
$$

By construction we have $\Omega_{0}=\mathcal{A}_{0}$ and $\Omega_{1}=\mathcal{A}_{1}$, while in general $\Omega_{p} \subset \mathcal{A}_{p}$. 
Let us define for any $p \geq 0$ the homologies of the digraph $G$ with coefficients from $\mathbb{K}$ by

$$
H_{p}(G, \mathbb{K})=H_{p}(G):=H_{p}\left(\Omega_{*}(G)\right)=\left.\operatorname{ker} \partial\right|_{\Omega_{p}} /\left.\operatorname{Im} \partial\right|_{\Omega_{p+1}} .
$$

Let us note that homology groups $H_{p}(G)$ (as well as the modules $\Omega_{p}(G)$ ) can be computed directly by definition using simple tools of linear algebra, in particular, those implemented in modern computational software. On the other hand, some theoretical tools for computation of homology groups like Künneth formulas were developed in [5].

Example 2.7. Consider a digraph $G$ as on Fig. 1. A direct computation

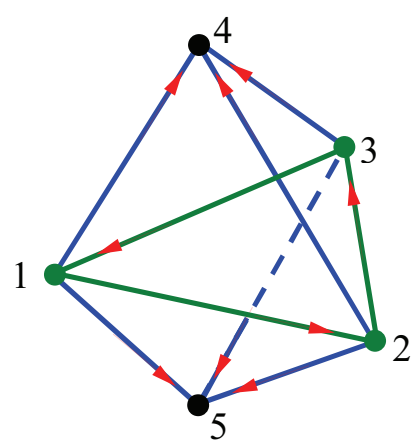

Figure 1: Planar digraph with a nontrivial homology group $H_{2}$

shows that $H_{1}(G, \mathbb{K})=\{0\}$ and $H_{2}(G, \mathbb{K}) \cong \mathbb{K}$, where $H_{2}(G)$ is generated by

$$
e_{124}+e_{234}+e_{314}-\left(e_{125}+e_{235}+e_{315}\right) .
$$

It is easy to see that $G$ is a planar graph but nevertheless its second homology group is non-zero. This shows that the digraph homologies "see" some nontrivial intrinsic dimensions of digraphs that are not necessarily related to embedding properties.

Example 2.8. Fix $n \geq 3$. Denote by $S_{n}$ a digraph with the vertex set $V_{S_{n}}=\{0, \ldots, n-1\}$ and with the set of edges $E_{S_{n}}$ that contains for any $i \in V_{S_{n}}$ exactly one of the edges $i \rightarrow i+1, i+1 \rightarrow i$ (where $n \equiv 0$ ), and no other edge. We refer to $S_{n}$ as a cycle digraph. 
The following 1-path on $S_{n}$

$$
\varpi=\sum_{\left\{i \in S_{n}: i \rightarrow i+1\right\}} e_{i(i+1)}-\sum_{\left\{i \in S_{n}: i+1 \rightarrow i\right\}} e_{(i+1) i}
$$

lies in $\Omega_{1}\left(S_{n}\right)$ and is closed. We will refer to $\varpi$ as a standard 1-path on $S_{n}$. It is possible to show that $\varpi$ generates the space of all closed 1-paths in $\Omega_{1}\left(S_{n}\right)$, which is therefore one-dimensional. The homology group $H_{1}\left(S_{n}, \mathbb{K}\right)$ is, hence, generated by the homology class $[\varpi]$, provided this class is nontrivial. One can show that $[\varpi]=0$ if and only if $S_{n}$ is isomorphic to one of the following two digraphs:

a triangle

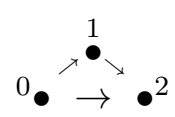
or a square

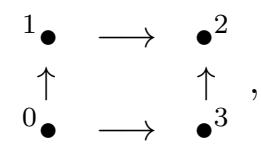

so that in this case $H_{1}\left(S_{n}, \mathbb{K}\right)=\{0\}$. In the case of triangle, $\varpi$ is the boundary of the 2-path $e_{012} \in \Omega_{2}$, and, in the case of square, $\varpi$ is the boundary of $e_{012}-e_{032} \in \Omega_{2}$. $\mathbb{K}$.

If $S_{n}$ is neither triangle nor square, then $[\varpi]$ is a generator of $H_{1}\left(S_{n}, \mathbb{K}\right) \cong$

Proposition 2.9. Let $G$ be any finite digraph. Then any $\omega \in \Omega_{2}(G, \mathbb{Z})$ can be represented as a linear combination of the $\partial$-invariant 2-paths of following three types:

1) $e_{i j i}$ with $i \rightarrow j \rightarrow i$ (a double edge in $\left.G\right)$;

2) $e_{i j k}$ with $i \rightarrow j \rightarrow k$ and $i \rightarrow k$ (a triangle as a subgraph of $G$ );

3) $e_{i j k}-e_{i m k}$ with $i \rightarrow j \rightarrow k, i \rightarrow m \rightarrow k, i \not \rightarrow k, i \neq k$ (a square as a subgraph of $G$ ).

Proof. Since the 2-path $\omega$ is allowed, it can be represented as a sum of elementary 2-path $e_{i j k}$ with $i \rightarrow j \rightarrow k$ multiplied with +1 or -1 . If $k=$ $i$ then $e_{i j k}$ is a double edge. If $i \neq k$ and $i \rightarrow k$ then $e_{i j k}$ is a triangle. Subtracting from $\omega$ all double edges and triangles, we can assume that $\omega$ has no such terms any more. Then, for any term $e_{i j k}$ in $\omega$ we have $i \neq k$ and $i \not \rightarrow k$. Fix such a pair $i, k$ and consider any vertex $j$ with $i \rightarrow j \rightarrow k$. The 1-path $\partial \omega$ is the sum of 1-paths of the form

$$
\partial e_{i j k}=e_{i j}-e_{i k}+e_{j k} .
$$


Since $\partial \omega$ is allowed but $e_{i k}$ is not allowed, the term $e_{i k}$ should cancel out after we sum up all such terms over all possible $j$. Therefore, the number of $j$ such that $e_{i j k}$ enters $\omega$ with coefficient +1 is equal to the number of $j$ such that $e_{i j k}$ enters in $\omega$ with the coefficient -1 . Combining the pair with +1 and -1 together, we obtain that $\omega$ is the sum of the terms of the third type (squares).

Theorem 2.10. Let $G$ and $G^{\prime}$ be two digraphs, and $f: G \rightarrow G^{\prime}$ be a digraph map. Then the map $\left.f_{*}\right|_{\Omega_{p}(G)}$ (where $f_{*}$ is the induced map (2.4)) provides a morphism of chain complexes

$$
\Omega_{*}(G, \mathbb{K}) \rightarrow \Omega_{*}\left(G^{\prime}, \mathbb{K}\right)
$$

and, consequently, a homomorphism of homology groups

$$
H_{*}(G, \mathbb{K}) \rightarrow H_{*}\left(G^{\prime}, \mathbb{K}\right)
$$

that will also be denoted by $f_{*}$.

Proof. By construction $\Omega_{p}(G)$ is a submodule of $\mathcal{R}_{p}(G)$, and all we need to prove is that

$$
f_{*}\left(\Omega_{p}(G)\right) \subset \Omega_{p}\left(G^{\prime}\right) .
$$

Let us first show that

$$
f_{*}\left(\mathcal{A}_{p}(G)\right) \subset \mathcal{A}_{p}\left(G^{\prime}\right) .
$$

It suffices to prove that if $e_{i_{0} \ldots i_{p}}$ is allowed on $G$ then $f_{*}\left(e_{i_{0} \ldots i_{p}}\right)$ is allowed on $G^{\prime}$. Indeed, if $e_{f\left(i_{0}\right) \ldots\left(i_{p}\right)}$ is irregular then we have by $(2.5)$ that $f_{*}\left(e_{i_{0} \ldots i_{p}}\right)=$ $0 \in \mathcal{A}_{p}\left(G^{\prime}\right)$. If $e_{f\left(i_{0}\right) \ldots\left(i_{p}\right)}$ is regular then $f\left(i_{k}\right) \neq f\left(i_{k+1}\right)$ for all $k=0, \ldots, p-$ 1. Since $i_{k} \rightarrow i_{k+1}$ on $G$, by the definition of a digraph map we have either $f\left(i_{k}\right) \rightarrow f\left(i_{k+1}\right)$ on $G^{\prime}$ or $f\left(i_{k}\right)=f\left(i_{k+1}\right)$. Since the second possibility is excluded, we obtain $f\left(i_{k}\right) \rightarrow f\left(i_{k+1}\right)$ for all $k$, whence it follows that $f_{*}\left(e_{i_{0} \ldots i_{p}}\right)=e_{f\left(i_{0}\right) \ldots\left(i_{p}\right)}$ is allowed on $G^{\prime}$.

Now we can prove (2.10). For any $v \in \Omega_{p}(G)$ we have by $(2.7) v \in \mathcal{A}_{p}(G)$ and $\partial v \in \mathcal{A}_{p-1}(G)$, whence

$$
f_{*}(v) \in \mathcal{A}_{p}\left(G^{\prime}\right) \text { and } \partial\left(f_{*}(v)\right)=f_{*}(\partial v) \in \mathcal{A}_{p-1}\left(G^{\prime}\right),
$$

which implies $f_{*}(v) \in \Omega_{p}\left(G^{\prime}\right)$. 


\subsection{Cylinders}

For any digraph $G$ consider its product $G \boxminus I$ with the digraph $I=\left({ }^{0} \bullet \rightarrow \bullet{ }^{1}\right)$ (see Definition 2.3).

Definition 2.11. The digraph $G \square I$ is called the cylinder over $G$ and will be denoted by $\mathrm{Cyl} G$ or by $\widehat{G}$.

By the definition of Cartesian product, the set of vertices of $\widehat{G}$ is $\widehat{V}=$ $V \times\{0,1\}$, and the set $\widehat{E}$ of its edges is defined by the rule: $(x, a) \rightarrow(y, b)$ if and only if either $x \rightarrow y$ in $G$ and $a=b$ or $x=y$ and $a \rightarrow b$ in $I$. We shall put the hat $\widehat{ }$ over all notation related to $\widehat{G}$, for example, $\widehat{\mathcal{R}}_{p}:=\mathcal{R}_{p}(\widehat{G})$ and $\widehat{\Omega}_{p}:=\Omega_{p}(\widehat{G})$. One can identify $\widehat{V}=V \times\{0,1\}$ with $V \sqcup V^{\prime}$ where $V^{\prime}$ is a copy of $V$, and use the notation $(x, 0) \equiv x$ and $(x, 1) \equiv x^{\prime}$.

Define the operation of lifting paths from $G$ to $\widehat{G}$ as follows. If $v=e_{i_{0} \ldots i_{p}}$ then $\widehat{v}$ is a $(p+1)$-path in $\widehat{G}$ defined by

$$
\widehat{v}=\sum_{k=0}^{p}(-1)^{k} e_{i_{0} \ldots i_{k} i_{k}^{\prime} \ldots i_{p}^{\prime}}
$$

By $\mathbb{K}$-linearity this definition extends to all $v \in \mathcal{R}_{p}$, thus giving $\widehat{v} \in \widehat{\mathcal{R}}_{p+1}$. It follows that, for any $v \in \mathcal{R}_{p}$ and any path $i_{0} \ldots i_{p}$ on $G$,

$$
\widehat{v}^{i_{0} \ldots i_{k} i_{k}^{\prime} \ldots i_{p}^{\prime}}=(-1)^{k} v^{i_{0} \ldots i_{p}}
$$

Clearly, $i_{0} \ldots i_{p}$ is allowed in $G$ if and only if $i_{0} \ldots i_{k} i_{k}^{\prime} \ldots i_{p}^{\prime}$ is allowed in $\widehat{G}$ :

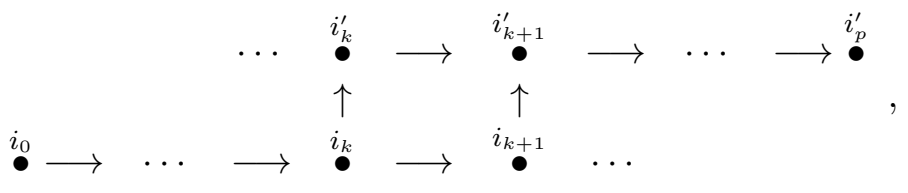

for some/all $k$. Hence, we see that $v \in \mathcal{A}_{p}$ if and only if $\widehat{v} \in \widehat{\mathcal{A}}_{p+1}$.

Proposition 2.12. If $v \in \Omega_{p}$ then $\widehat{v} \in \widehat{\Omega}_{p+1}$.

Proof. We need to prove that if $v \in \mathcal{A}_{p}$ and $\partial v \in \mathcal{A}_{p-1}$ then $\partial \widehat{v} \in \widehat{\mathcal{A}}_{p}$. Let us prove first some properties of the lifting. For any path $v$ in $G$ define its 
image $v^{\prime}$ in $G^{\prime}=\left(V^{\prime}, E^{\prime}\right)$ by

$$
\left(e_{i_{0} \ldots i_{p}}\right)^{\prime}=e_{i_{0}^{\prime} \ldots i_{p}^{\prime}}
$$

Let us show first that, for any $p$-path $u$ and $q$-path $v$ on $G$, the following identity holds:

$$
\widehat{u v}=\widehat{u} v^{\prime}+(-1)^{p+1} u \widehat{v}
$$

It suffices to prove it for $u=e_{i_{0} \ldots i_{p}}$ and $v=e_{j_{0} \ldots j_{q}}$. Then $u v=e_{i_{0} \ldots i_{p} j_{0} \ldots j_{q}}$ and

$$
\begin{aligned}
\widehat{u v} & =\sum_{k=0}^{p}(-1)^{k} e_{i_{0} \ldots i_{k} i_{k}^{\prime} \ldots i_{p}^{\prime} j_{0}^{\prime} \ldots j_{q}^{\prime}}+\sum_{k=0}^{q}(-1)^{k+p+1} e_{i_{0} \ldots i_{p} j_{0} \ldots j_{k} j_{k}^{\prime} \ldots j_{q}^{\prime}} \\
& =\widehat{u} v^{\prime}+(-1)^{p+1} u \widehat{v} .
\end{aligned}
$$

Now let us show that, for any $p$-path $v$ with $p \geq 0$

$$
\partial \widehat{v}=-\widehat{\partial v}+v^{\prime}-v \text {. }
$$

It suffices to prove it for $v=e_{i_{0} \ldots i_{p}}$, which will be done by induction in $p$. For $p=0$ write $v=e_{a}$ so that $\partial v=0$ and $\widehat{v}=e_{a a^{\prime}}$ whence

$$
\partial \widehat{v}=e_{a^{\prime}}-e_{a}=-\widehat{\partial v}+v^{\prime}-v .
$$

For $p>1$ write $v=u e_{i_{p}}$ where $u=e_{i_{0} \ldots i_{p-1}}$. Using (2.13) and the inductive hypothesis with the $(p-1)$-path $u$ we obtain

$$
\begin{aligned}
\partial \widehat{v} & =\partial\left(\widehat{u} e_{i_{p}^{\prime}}+(-1)^{p} u e_{i_{p} i_{p}^{\prime}}\right) \\
& =(\partial \widehat{u}) e_{i_{p}^{\prime}}+(-1)^{p+1} \widehat{u}+(-1)^{p}(\partial u) e_{i_{p} i_{p}^{\prime}}+u\left(e_{i_{p}^{\prime}}-e_{i_{p}}\right) \\
& =\left(-\widehat{\partial u}+u^{\prime}-u\right) e_{i_{p}^{\prime}}+(-1)^{p+1} \widehat{u}+(-1)^{p}(\partial u) e_{i_{p} i_{p}^{\prime}}+u e_{i_{p}^{\prime}}-v \\
& =-(\widehat{\partial u}) e_{i_{p}^{\prime}}+v^{\prime}+(-1)^{p+1} \widehat{u}+(-1)^{p}(\partial u) e_{i_{p} i_{p}^{\prime}}-v .
\end{aligned}
$$

On the other hand,

$$
\widehat{\partial v}=\left((\partial u) e_{i_{p}}+(-1)^{p} u\right)^{\wedge}=(\widehat{\partial u}) e_{i_{p}^{\prime}}+(-1)^{p-1}(\partial u) e_{i_{p} i_{p}^{\prime}}+(-1)^{p} \widehat{u}
$$

whence it follows that $\partial \widehat{v}+\widehat{\partial v}=v^{\prime}-v$, which finishes the proof of (2.14).

Finally, if $v \in \mathcal{A}_{p}$ and $\partial v \in \mathcal{A}_{p-1}$ then $v^{\prime}$ and $\widehat{\partial v}$ belong to $\widehat{\mathcal{A}}_{p}$ whence it follows from (2.14) also $\partial \widehat{v} \in \widehat{\mathcal{A}}_{p}$. This proves that $\widehat{v} \in \widehat{\mathcal{A}}_{p+1}$. 
Example 2.13. The cylinder over the digraph ${ }^{0} \bullet \rightarrow \bullet^{1}$ is a square

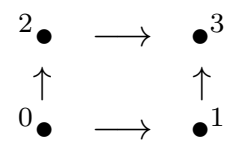

Lifting a $\partial$-invariant 1-path $e_{01} \in \Omega_{1}$ we obtain a $\partial$-invariant 2-path on the square: $e_{00^{\prime} 1^{\prime}}-e_{011^{\prime}}$, that can be rewritten in the form $e_{023}-e_{013}$.

The cylinder over a square is a 3 -cube that is shown in Fig. 2.

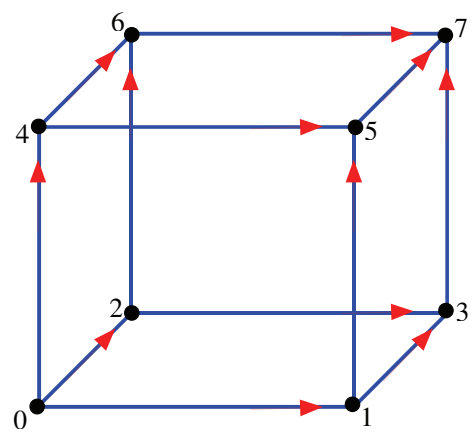

Figure 2: 3-cube

Lifting the 2-path $e_{023}-e_{013}$ we obtain a $\partial$-invariant 3 -path on the 3 cube:

$$
e_{0467}-e_{0267}+e_{0237}-e_{0457}+e_{0157}-e_{0137}
$$

Defining further $n$-cube as the cylinder over $(n-1)$-cube, we see that $n$-cube determines a $\partial$-invariant $n$-path that is a lifting of a $\partial$-invariant $(n-1)$-path from $(n-1)$-cube and that is an alternating sum of $n$ ! elementary terms. One can show that this $n$-path generates $\Omega_{n}$ on $n$-cube (see [7]).

\section{Homotopy theory of digraphs}

In this Section we introduce a homotopy theory of digraphs and establish the relations between this theory and the homology theory of digraphs of [5] and [7]. 


\subsection{The notion of homotopy}

Fix $n \geq 0$. Denote by $I_{n}$ any digraph whose the set of vertices is $\{0,1, \ldots, n\}$ and the set of edges contains exactly one of the edges $i \rightarrow(i+1),(i+1) \rightarrow i$ for any $i=0,1, \ldots, n-1$, and no other edges. A digraph $I_{n}$ is called a line digraph. Denote by $\mathcal{I}_{n}$ the set of all line digraphs $\mathcal{I}_{n}$ and by $\mathcal{I}$ the union of all $\mathcal{I}_{n}$.

Clearly, there is only one digraph in $\mathcal{I}_{0}$ - the one-point digraph. There are two digraphs in $\mathcal{I}_{1}$ : the digraph $I$ with the edge $(0 \rightarrow 1)$ and the digraph $I^{-}$with the edge $(1 \rightarrow 0)$.

Definition 3.1. Let $G, H$ be two digraphs. Two digraph maps $f, g: G \rightarrow H$ are called homotopic if there exists a line digraph $I_{n} \in \mathcal{I}_{n}$ with $n \geq 1$ and a digraph map

$$
F: G \boxminus I_{n} \rightarrow H
$$

such that

$$
\left.F\right|_{G \triangleright\{0\}}=f \text { and }\left.F\right|_{G \sqcup\{n\}}=g
$$

In this case we shall write $f \simeq g$. The map $F$ is called a homotopy between $f$ and $g$.

In the case $n=1$ we refer to the map $F$ as an one-step homotopy between $f$ and $g$. In this case the identities (3.1) determine $F$ uniquely, and the requirement is that the so defined $F$ is a digraph map of $G \square I_{1}$ to $H$. Since for $I_{1}$ there are only two choices $0 \rightarrow 1$ and $0 \leftarrow 1$, we obtain that $f$ and $g$ are one-step homotopic, if

(3.2) either $f(x) \rightrightarrows g(x)$ for all $x \in V_{G}$ or $g(x) \rightrightarrows f(x)$ for all $x \in V_{G}$.

It follows that $f$ and $g$ are homotopic if there is a finite sequence of digraph maps $f=f_{0}, f_{1}, \ldots, f_{n}=g$ from $G$ to $H$ such that $f_{k}$ and $f_{k+1}$ are one-step homotopic. It is obvious that the relation " $\simeq$ " is an equivalence relation on the set of all digraph maps from $G$ to $H$.

Definition 3.2. Two digraphs $G$ and $H$ are called homotopy equivalent if there exist digraph maps

$$
f: G \rightarrow H, \quad g: H \rightarrow G
$$


such that

$$
f \circ g \simeq \operatorname{id}_{H}, \quad g \circ f \simeq \operatorname{id}_{G}
$$

In this case we shall write $H \simeq G$. The maps $f$ and $g$ as in (3.4) are called homotopy inverses of each other.

A digraph $G$ is called contractible if $G \simeq\{*\}$ where $\{*\}$ is a single vertex digraph. It follows from Definition 3.2 that a digraph $G$ is contractible if and only if there is a digraph map $h: G \rightarrow G$ such that the image of $h$ consists of a single vertex and $h \simeq \operatorname{id}_{G}$. Examples of contractible digraphs will be given in Section 3.3.

\subsection{Homotopy preserves homologies}

Now we can prove the first result about connections between homotopy and homology theories for digraphs.

Theorem 3.3. Let $G, H$ be two digraphs.

(i) Let $f \simeq g: G \rightarrow H$ be two homotopic digraph maps. Then these maps induce the identical homomorphisms of homology groups of $G$ and $H$, that is, the maps

$$
f_{*}: H_{p}(G) \rightarrow H_{p}(H) \quad \text { and } \quad g_{*}: H_{p}(G) \rightarrow H_{p}(H)
$$

are identical.

(ii) If the digraphs $G$ and $H$ are homotopy equivalent, then they have isomorphic homology groups. Furthermore, if the homotopical equivalence of $G$ and $H$ is provided by the digraph maps (3.3) then their induced maps $f_{*}$ and $g_{*}$ provide mutually inverse isomorphisms of the homology groups of $G$ and $H$.

In particular, if a digraph $G$ is contractible, then all the homology groups of $G$ are trivial, except for $H_{0}$.

Proof. $(i)$ Let $F$ be a homotopy between $f$ and $g$ as in Definition 3.1. Consider first the case $n=1$ and let $I_{n}$ be the digraph $I=(0 \rightarrow 1)$ (the case 
$I_{n}=I^{-}$is similar). The maps $f$ and $g$ induce morphisms of chain complexes

$$
f_{*}, g_{*}: \Omega_{*}(G) \rightarrow \Omega_{*}(H)
$$

and $F$ induces a morphism

$$
F_{*}: \Omega_{*}(G \boxminus I) \rightarrow \Omega_{*}(H)
$$

Note that, for any path $v \in \Omega_{*}(G \boxminus I)$ that lies in $G \boxminus\{0\}$, we have $F_{*}(v)=$ $f_{*}(v)$, and for any path $v^{\prime} \in \Omega_{*}(G \boxminus I)$ that lies in $G \boxminus\{1\}$, we have $F_{*}\left(v^{\prime}\right)=$ $g\left(v^{\prime}\right)$.

In order to prove that $f_{*}$ and $g_{*}$ induce the identical homomorphisms $H_{*}(G) \rightarrow H_{*}(H)$, it suffices by [10, Theorem 2.1, p.40] to construct a chain homotopy between the chain complexes $\Omega_{*}(G)$ and $\Omega_{*}(H)$, that is, the $\mathbb{K}$ linear mappings

$$
L_{p}: \Omega_{p}(G) \rightarrow \Omega_{p+1}(H)
$$

such that

$$
\partial L_{p}+L_{p-1} \partial=g_{*}-f_{*}
$$

(note that all the terms here are mapping from $\Omega_{p}(G)$ to $\Omega_{p}(H)$ ). Let us define the mapping $L_{p}$ as follows

$$
L_{p}(v)=F_{*}(\widehat{v})
$$

for any $v \in \Omega_{p}(G)$, where $\widehat{v} \in \Omega_{p+1}(G \boxminus I)$ is lifting of $v$ to the graph $\widehat{G}=$ $G \boxminus I$ defined in Section 2.5. Using $\partial F_{*}=F_{*} \partial$ (see Theorem 2.10) and the product rule (2.14), we obtain

$$
\begin{aligned}
\left(\partial L_{p}+L_{p-1} \partial\right)(v) & =\partial\left(F_{*}(\widehat{v})\right)+F_{*}(\widehat{\partial v}) \\
& =F_{*}(\partial \widehat{v})+F_{*}(\widehat{\partial v}) \\
& =F_{*}(\partial \widehat{v}+\widehat{\partial v}) \\
& =F_{*}\left(v^{\prime}-v\right) \\
& =g_{*}(v)-f_{*}(v) .
\end{aligned}
$$

The case of an arbitrary $n$ follows then by induction. 
(ii) Let $f, g$ be the maps from Definition 3.2. Then they induce the following mappings

$$
H_{p}(G) \stackrel{f_{*}}{\rightarrow} H_{p}(H) \stackrel{g_{*}}{\rightarrow} H_{p}(G) \stackrel{f_{*}}{\rightarrow} H_{p}(H) .
$$

By $(i)$ and (3.4) we have $f_{*} \circ g_{*}=\mathrm{id}$ and $g_{*} \circ f_{*}=\mathrm{id}$, which implies that $f_{*}$ and $g_{*}$ are mutually inverse isomorphisms of $H_{p}(G)$ and $H_{p}(H)$.

\subsection{Retraction}

A (induced) sub-digraph $H$ of a digraph $G$ is a digraph whose set of vertices is a subset of that of $G$ and the edges of $H$ are all those edges of $G$ whose adjacent vertices belong to $H$.

Definition 3.4. Let $G$ be a digraph and $H$ be its sub-digraph.

(i) A retraction of $G$ onto $H$ is a digraph map $r: G \rightarrow H$ such that $\left.r\right|_{H}=\mathrm{id}_{H}$.

(ii) A retraction $r: G \rightarrow H$ is called a deformation retraction if $i \circ r \simeq$ $\operatorname{id}_{G}$, where $i: H \rightarrow G$ is the natural inclusion map.

Proposition 3.5. Let $r: G \rightarrow H$ be a deformation retraction. Then $G \simeq H$ and the maps $r, i$ are homotopy inverses.

Proof. By definition of retraction we have $r \circ i=\operatorname{Id}_{H}$ and, in particular $r \circ i \simeq \operatorname{id}_{H}$. Since $i \circ r \simeq \operatorname{id}_{G}$, we obtain by Definition 3.2 that $G \simeq H$.

In general the existence of a deformation retraction $r: G \rightarrow H$ is a stronger condition that the homotopy equivalence $G \simeq H$. However, in the case when $H=\{*\}$, the existence of a deformation retraction $r: G \rightarrow\{*\}$ is equivalent to the contractibility of $G$, which follows from the remark after Definition 3.2.

The next two statements provide a convenient way of constructing a deformation retraction.

Proposition 3.6. Let $r: G \rightarrow H$ be a retraction of a digraph $G$ onto a subdigraph H. Assume that there exists a finite sequence $\left\{f_{k}\right\}_{k=0}^{n}$ of digraph maps $f_{k}: G \rightarrow G$ with the following properties:

1) $f_{0}=\operatorname{id}_{G}$;

2) $f_{n}=i \circ r$ (where $i$ is the inclusion map $i: H \rightarrow G$ ), that is, $f_{n}(v)=$ $r(v)$ for all vertices $v$ of $G$; 
3) for any $k=1, \ldots, n$ either $f_{k-1}(x) \rightrightarrows f_{k}(x) \forall x \in V_{G}$ or $f_{k}(x) \rightrightarrows f_{k-1}(x)$ $\forall x \in V_{G}$.

Then $r$ is a deformation retraction, the digraphs $G$ and $H$ are homotopy equivalent, and $i, r$ are their homotopy inverses.

Proof. Since $f_{k-1}$ and $f_{k}$ satisfy (3.2), we see that $f_{k-1} \simeq f_{k}$ whence by induction we obtain that $f_{n} \simeq f_{0}$ and, hence, $i \circ r \simeq \operatorname{id}_{G}$. Therefore, $r$ is a deformation retraction, and the rest follow from Proposition 3.4.

Corollary 3.7. Let $r: G \rightarrow H$ be a retraction of a digraph $G$ onto a subdigraph $H$ and

$$
x \rightrightarrows r(x) \text { for all } x \in V_{G} \text { or } \quad r(x) \rightrightarrows x \text { for all } x \in V_{G}
$$

Then $r$ is a deformation retraction, the digraphs $G$ and $H$ are homotopy equivalent, and $i, r$ are their homotopy inverses.

Clearly, Corollary 3.7 is an important particular case $n=1$ of Proposition 3.6. Note also that the condition (3.5) is automatically satisfies for all $x \in V_{H}$, so in applications it remains to verify it for $v \in V_{G} \backslash V_{H}$.

Corollary 3.8. For any digraph $G$ and for any line digraph $I_{n} \in \mathcal{I}_{n}(n \geq 0)$ we have $G \boxminus I_{n} \simeq G$.

Proof. It suffices to show that $G \boxminus I_{n} \simeq G \boxminus I_{n-1}$ where $I_{n-1}$ is obtained from $I_{n}$ by removing the vertex $n$ and the adjacent edge, and then to argue by induction since $G \boxminus I_{0}=G$. Define a retraction $r: G \boxminus I_{n} \rightarrow G \boxminus I_{n-1}$ by

$$
r(x, k)= \begin{cases}(x, k), & k \leq n-1, \\ (x, n-1), & k=n .\end{cases}
$$

Let us show that $r$ is an 1-step deformation retraction, that is, $r$ satisfied $(3.5)$ :

$$
(x, k) \rightrightarrows r(x, k) \forall(x, k) \in G \boxminus I_{n} \quad \text { or } \quad r(x, k) \rightrightarrows(x, k) \forall(x, k) \in G \boxminus I_{n}
$$

Indeed, for $k \leq n-1$ this is obvious. If $k=n$ then consider two cases.

1) If $(n-1) \rightarrow n$ in $I_{n}$ then $(x, n-1) \rightarrow(x, n)$ in $G \square I_{n}$ whence

$$
r(x, k)=r(x, n)=(x, n-1) \rightarrow(x, n)=(x, k) .
$$


2) If $n \rightarrow(n-1)$ in $I_{n}$ then $(x, n) \rightarrow(x, n-1)$ in $G \boxminus I_{n}$ whence

$$
(x, k) \rightarrow r(x, k)
$$

Corollary 3.9. Let $G$ be a digraph. Fix some $n \in \mathbb{N}$ and consider for any $k=0, \ldots, n$ the natural inclusion

$$
i_{k}: G \rightarrow G \boxminus I_{n}, \quad i_{k}(v)=(v, k)
$$

and a natural projection

$$
p: G \boxminus I_{n} \rightarrow G, \quad p(v, k)=v
$$

Then the maps i,p induce isomorphism of homology groups.

Proof. The projection $p$ can be decomposed into composition of retractions $G \boxminus I_{m} \rightarrow G \boxminus I_{m-1}$ which are homotopy equivalences by the proof of Corollary 3.8. Therefore, $p$ is also a homotopy equivalence and hence induces isomorphism of homology groups. The inclusion $i_{k}$ can be decomposed into composition of natural inclusions $G \bullet I_{m-1} \rightarrow G \boxminus I_{m}$, each of them being homotopy inverse of the retraction $G \boxminus I_{m} \rightarrow G \boxminus I_{m-1}$, which implies the claim.

Example 3.10. A digraph $G$ is called a tree if the underlying undirected graph is a tree. We claim that if a digraph $G$ is a connected tree then $G$ is contractible. Indeed, let $a$ be a pendant vertex of $G$ and let $b$ be another vertex such that $a \rightarrow b$ or $b \leftarrow a$. Let $G^{\prime}$ be the subgraph of $G$ that is obtained from $G$ by removing the vertex $a$ with the adjacent edge. Then the map $r$ : $G \rightarrow G^{\prime}$ defined by $r(a)=b$ and $\left.r\right|_{H}=$ id is by Corollary 3.7 a deformation retraction, whence $G \simeq G^{\prime}$. Since $G^{\prime}$ is also a connected tree, continuing the procedure of removing of a pendant vertices, we obtain in the end that $G$ is contractible.

Example 3.11. A digraph $G$ is called star-like (resp. inverse star-like) if there is a vertex $a \in V_{G}$ such that $a \rightarrow x(\operatorname{resp} . x \rightarrow a)$ for all $x \in V_{G} \backslash$ $\{a\}$. if $G$ is a (inverse) star-like digraph, then the map $r: G \rightarrow\{a\}$ is by Corollary 3.7 a deformation retraction, whence we obtain $G \simeq\{a\}$, that is, $G$ is contractible. Consequently, all homology groups of $G$ are trivial except for $H_{0}$. 
For example, consider a digraph-simplex of dimension $n$, which is a digraph $G$ with the set of vertices $\{0,1, \ldots, n\}$ and the set of edges given by the condition

$$
i \rightarrow j \Longleftrightarrow i<j
$$

(a digraph-simplex with $n=3$ is shown on the left panel on Fig. 3). Then $G$ is star-like and, hence, $G$ is contractible. In particular, the triangular digraph from Example 2.8 is contractible. Another star-like digraph is shown on the right panel of Fig. 3 .
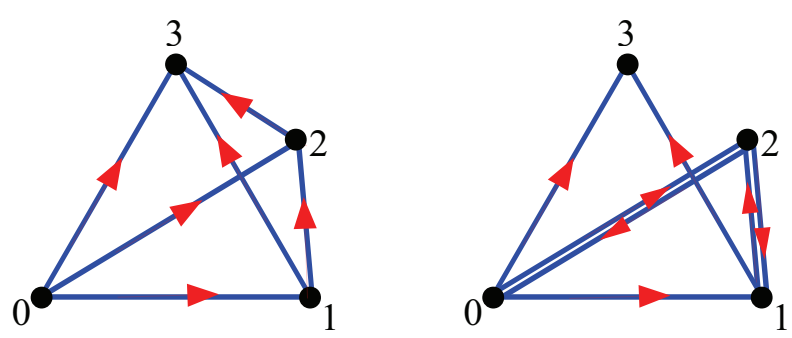

Figure 3: Star-like digraphs

Example 3.12. For any $n \geq 1$, consider the $n$-dimensional cube

$$
I^{n}=\underbrace{I \boxminus I \boxminus \cdots \boxminus I}_{n \text { times }}
$$

For example, $I_{2}$ is the square from Example 2.8 and $I_{3}$ is a 3-cube shown on Fig. 2. By Corollary 3.8 we have $I^{k} \simeq I^{k-1}$, whence we obtain that, for all $n, I^{n} \simeq I$, which implies that $I^{n}$ is contractible. In particular, this applies to a square digraph from Example 2.8. Consequently, the all homology groups of $I^{n}$ are trivial except for $H_{0}$.

Example 3.13. Let $S_{n}$ be a cycle digraph from Example 2.8. If $S_{n}$ is the triangle or square as in (2.9) then $S_{n}$ is contractible as was shown in Examples 3.11 and 3.12 , respectively. If $S_{n}$ is neither triangle nor square then by Example $2.8 H_{1}\left(S_{n}, \mathbb{K}\right) \cong \mathbb{K}$ and, hence, $S_{n}$ is not contractible. In particular, this is always the case when $n \geq 5$. Here are other examples of 
non-contractible cycles with $n=3,4$ :

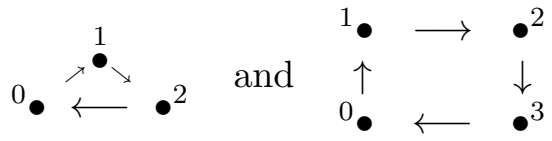

Let us show that two cycles $S_{n}$ and $S_{m}$ with $n \neq m$ are not homotopy equivalent, except for the case when one of them is a triangle and the other is a square. Assume that $S_{n}$ and $S_{m}$ with $n<m$ are homotopy equivalent. Then by Theorem 3.3 there is a digraph map $f: S_{n} \rightarrow S_{m}$ such that $f_{*}: H_{1}\left(S_{n}\right) \rightarrow H_{1}\left(S_{m}\right)$ is an isomorphism. If homology groups $H_{1}\left(S_{n}\right)$ and $H_{1}\left(S_{m}\right)$ are not isomorphic then we are done. If they are isomorphic, then they are isomorphic to $\mathbb{K}$. Let $\varpi_{n} \in \Omega_{1}\left(S_{n}\right)$ be the generator of closed 1paths on $S_{n}$ and $\varpi_{m} \in \Omega_{1}\left(S_{m}\right)$ be the generator of closed 1-paths on $S_{n}$, as in $(2.8)$. Then $\left[\varpi_{n}\right]$ generates $H_{1}\left(S_{n}\right),\left[\varpi_{m}\right]$ generates $H_{1}\left(S_{m}\right)$, and we should have

$$
f_{*}\left(\left[\varpi_{n}\right]\right)=k\left[\varpi_{m}\right]
$$

for some non-zero constant $k \in \mathbb{K}$. Consequently, we obtain

$$
f_{*}\left(\varpi_{n}\right)=k \varpi_{m}
$$

which is impossible because $f$ cannot be surjective by $n<m$, whereas $\varpi_{m}$ uses all the vertices of $S_{m}$.

Example 3.14. Consider the digraph $G$ as on Fig. 4.

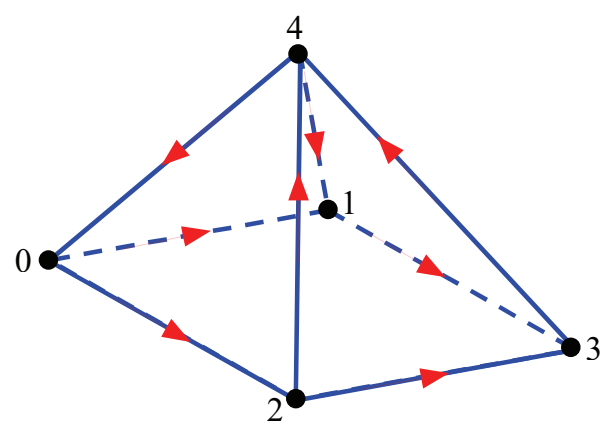

Figure 4: The digraph admits a deformation retraction onto a subgraph $\{1,3,4\}$ 
Consider also its sub-digraph $H$ with the vertex set $V_{H}=\{1,3,4\}$ and a retraction $r: G \rightarrow H$ given by $r(0)=1, r(2)=3$ and $\left.r\right|_{H}=$ id. By Corollary 3.7, $r$ is a deformation retraction, whence $G \simeq H$. Consequently, we obtain $H_{1}(G, \mathbb{K}) \cong H_{1}(H, \mathbb{K}) \cong \mathbb{K}$ and $H_{p}(G, \mathbb{K})=\{0\}$ for $p \geq 2$.

Example 3.15. Let $a$ be a vertex in a digraph $G$ and let $b_{0}, b_{1}, \ldots, b_{n}$ be all the neighboring vertices of $a$ in $G$. Assume that the following condition is satisfied:

$$
\forall i=1, \ldots, n \quad a \rightarrow b_{i} \Rightarrow b_{0} \rightarrow b_{i} \quad \text { and } \quad a \leftarrow b_{i} \Rightarrow b_{0} \leftarrow b_{i} .
$$

Denote by $H$ the digraph that is obtained from $G$ by removing a vertex $a$ with all adjacent edges. The map $r: G \rightarrow H$ given by $r(a)=b_{0}$ and $\left.r\right|_{H}=$ id is by Corollary 3.7 a deformation retraction, whence we obtain that $G \simeq H$. Consequently, all homology groups of $G$ and $H$ are the same. This is very similar to the results about transformations of simplicial complexes in the simple homotopy theory (see, for example, $[3]$ ).

In particular, (3.6) is satisfied if $a \rightarrow b_{i}$ and $b_{0} \rightarrow b_{i}$ for all $i \geq 1$ or $a \leftarrow$ $b_{i}$ and $b_{0} \leftarrow b_{i}$ for all $i \geq 1$. Two examples when (3.6) is satisfied are shown in the following diagram:
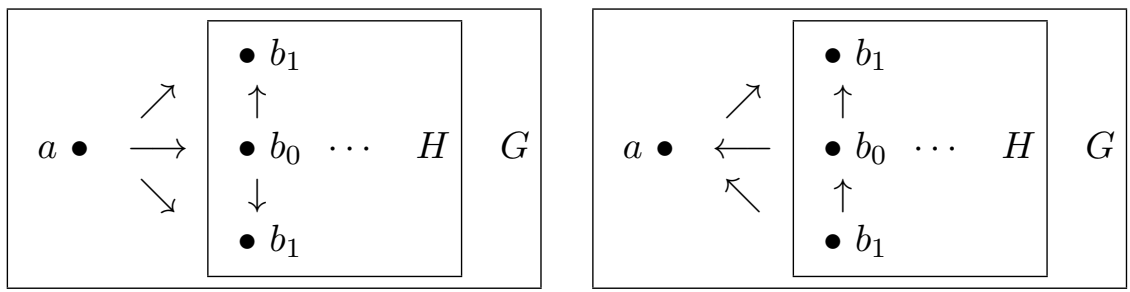

On the contrary, the digraph $G$ on following diagram

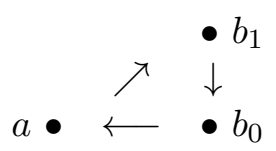

does not satisfy (3.6). Moreover, this digraph is not homotopy equivalent to $H=\left({ }^{0} \bullet \bullet^{1}\right.$ ) since $G$ and $H$ have different homology group $H_{1}$ (cf. Example 2.8).

For example, the digraph on the left panel of Fig. 5 is contractible as one can successively remove the vertices $5,4,3,2$ each time satisfying (3.6).

The digraph on the right panel of Fig. 5 is different from the left one only by the direction of the edge between 1 and 3, but it is not contractible as its $H_{2}$ group is non-trivial by Example 2.7. 

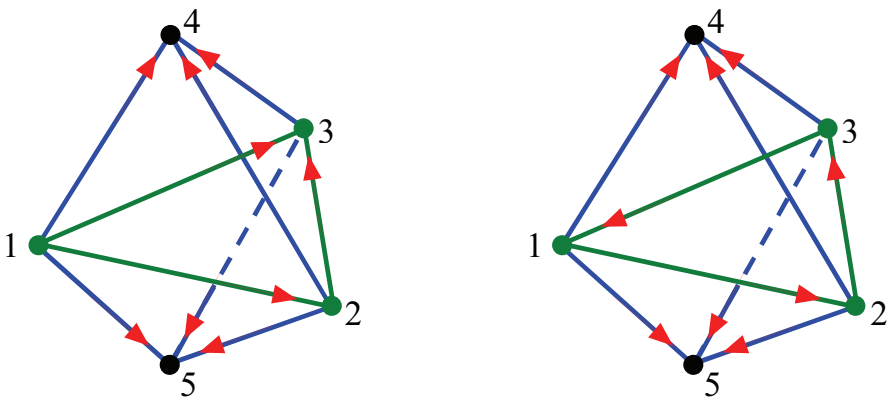

Figure 5: The left digraph is contractible while the right one is not.

Consider one more example: the digraph $G$ on Fig. 6.

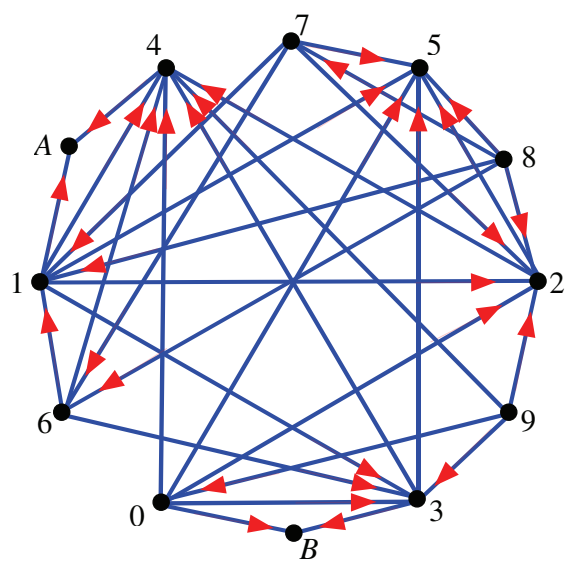

Figure 6: Digraph $G$ whose $H_{2}$ group is generated by an octahedron

Removing successively the vertices $A, B, 8,9,6,7$, which each time satisfy (3.6), we obtain a digraph $H$ with $V_{H}=\{0,1,2,3,4,5\}$ that is homotopy equivalent to $G$ and, in particular, has the same homologies as $G$. The digraph $H$ is shown in two ways on Fig. 7. Clearly, the second representation of this graph is reminiscent of an octahedron.

It is possible to show that $H_{p}(H, \mathbb{K})=\{0\}$ for $p=1$ and $p>2$ while $H_{2}(H, \mathbb{K}) \cong \mathbb{K}$. It follows that the same is true for the homology groups of $G$. Furthermore, it is possible to show that $H_{2}(G, \mathbb{K})$ is generated by the 

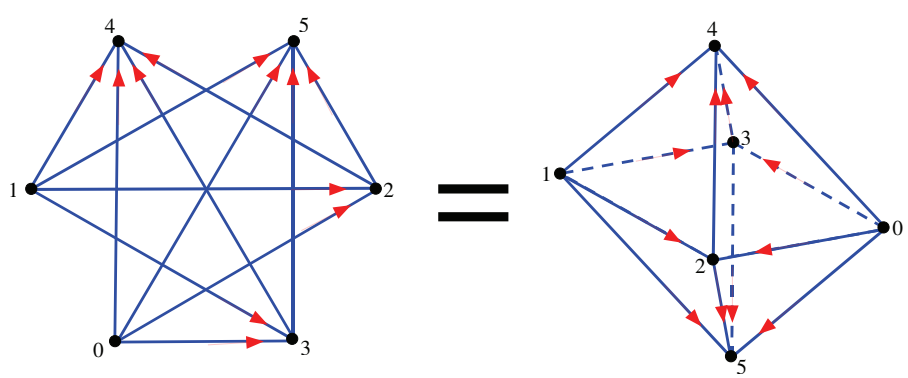

Figure 7: Two representations of the digraph $H$

following 2-path

$$
\omega=e_{024}-e_{025}-e_{034}+e_{035}-e_{124}+e_{125}+e_{134}-e_{135}
$$

that determines a 2-dimensional "hole" in $G$ given by the octahedron $H$. Note that on Fig. 6 this octahedron is hardy visible.

\subsection{Cylinder of a map}

Let us give some further examples of homotopy equivalent digraphs.

Definition 3.16. Let $G=\left(V_{G}, E_{G}\right)$ and $H=\left(V_{H}, E_{H}\right)$ be two digraphs and $f$ be a digraph map from $G$ to $H$. The cylinder $\mathrm{C}_{f}$ of $f$ is the digraph with the set of vertices $V_{\mathrm{C}_{f}}=V_{G} \sqcup V_{H}$ and with the set of edges $E_{\mathrm{C}_{f}}$ that consists of all the edges from $E_{G}$ and $E_{H}$ as well as of the edges of the form $x \rightarrow f(x)$ for all $x \in V_{G}$.

The inverse cylinder $\mathrm{C}_{f}^{-}$is defined in the same way except that the edge $x \rightarrow f(x)$ is replaced by $f(x) \rightarrow x$.

For example, for $f=\mathrm{id}_{G}$ we have $\mathrm{C}_{f}=G \boxminus I$ where $I=\left({ }^{0} \bullet \longrightarrow \bullet^{1}\right)$ and $\mathrm{C}_{f}^{-}=G \boxminus I^{-}$where $I^{-}=\left({ }^{0} \bullet \longleftarrow \bullet^{1}\right)$.

Example 3.17. Let $G$ be the digraph with vertices $\{0,1,2,3,4,5\}$ and $H$ is be the digraph with vertices $\{a, b, c\}$ as on Fig. 8. Consider the digraph map $f: G \rightarrow H$ given by $f(0)=f(1)=a, f(2)=f(3)=b$ and $f(4)=f(5)=$ c. The cylinder $\mathrm{C}_{f}$ of $f$ is shown on Fig. 8 . 


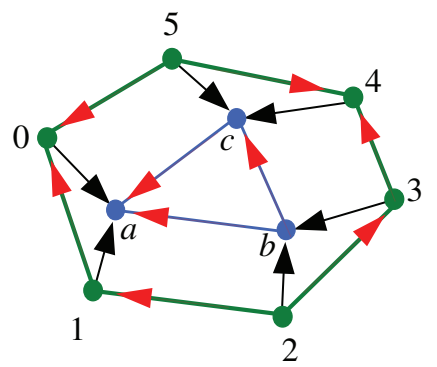

Figure 8: The cylinder of the map

Proposition 3.18. Let $f$ be a digraph map from $G$ to $H$. Then we have the following homotopy equivalences of the digraphs

$$
\mathrm{C}_{f} \simeq H \simeq \mathrm{C}_{f}^{-}
$$

Proof. The projection $p: \mathrm{C}_{f} \rightarrow H$ defined by

$$
p(x)= \begin{cases}x, & x \in V_{H}, \\ f(x), & x \in V_{G},\end{cases}
$$

is clearly an 1-step deformation retraction of $\mathrm{C}_{f}$ onto $H$, whence it follows by Corollary 3.7 that $\mathrm{C}_{f} \simeq H$. The case of the inverse cylinder $\mathrm{C}_{f}^{-}$is similar.

\section{Homotopy groups of digraphs}

In this Section we define homotopy groups of digraphs and describe theirs basic properties. For that, we introduce the concept of path-map in a digraph $G$, and then define a fundamental group of $G$. Then the higher homotopy group can be defined inductively as the fundamental group of the corresponding iterated loop-digraph.

A based digraph $G^{*}$ is a digraph $G$ with a fixed base vertex $* \in V_{G}$. A based digraph map $f: G^{*} \rightarrow H^{*}$ is a digraph map $f: G \rightarrow H$ such that $f(*)=*$. A category of based digraphs will be denoted by $\mathcal{D}^{*}$.

A homotopy between two based digraph maps $f, g: G^{*} \rightarrow H^{*}$ is defined as in Definition 3.1 with additional requirement that $\left.F\right|_{\{*\} \bullet I_{n}}=*$. 


\subsection{Construction of $\pi_{0}$}

Let $G^{*}$ be a based digraph, and $V_{2}^{*}=\{0,1\}$ be the based digraph consisting of two vertices, no edges and with the base vertex $0=*$. Let $\operatorname{Hom}\left(V_{2}^{*}, G^{*}\right)$ be the set of based digraph maps from $V_{2}^{*}$ to $G^{*}$. Note that the set of such maps is in one to one correspondence with the set of vertices of the digraph $G$.

Definition 4.1. We say that two digraph maps $\phi, \psi \in \operatorname{Hom}\left(V_{2}^{*}, G^{*}\right)$ are equivalent and write $\phi \simeq \psi$ if there exists $I_{n} \in \mathcal{I}$ and a digraph map

$$
f: I_{n} \rightarrow G,
$$

such that $f(0)=\phi(1)$ and $f(n)=\psi(1)$. The relation $\simeq$ is evidently an equivalence relation, and we denote by $[\phi]$ the equivalence class of the element $\phi$, and by $\pi_{0}\left(G^{*}\right)$ the set of classes of equivalence with the base point $*$ given by a class of equivalence of the trivial map $V_{2} \rightarrow * \in G$.

The set $\pi_{0}\left(G^{*}\right)$ coincides with the set of connected components of the digraph $G$. In particular, the digraph $G^{*}$ connected if $\pi_{0}\left(G^{*}\right)=*$.

Proposition 4.2. Any based digraph map $f: G^{*} \rightarrow H^{*}$ induces a map

$$
\pi_{0}(f): \pi_{0}\left(G^{*}\right) \rightarrow \pi_{0}\left(H^{*}\right)
$$

of based sets. The homotopic maps induce the same map of based sets. We have a functor from the category $\mathcal{D}^{*}$ of digraphs to the category based sets.

Proof. Let $x=[\phi] \in \pi_{0}\left(G^{*}\right)$ be presented by a digraph map $\phi: V_{2}^{*} \rightarrow G^{*}$ we put $y=\left[\pi_{0}(f)\right](x)=[f \circ \phi] \in \pi_{0}\left(H^{*}\right)$. It is an easy exercise to check that this map $\pi_{0}(f)$ is well defined and for the homotopic maps $f \simeq g: G^{*} \rightarrow H^{*}$ we have $\pi_{0}(f)=\pi_{0}(g)$.

\section{2. $C$-homotopy and $\pi_{1}$}

For any line digraph $I_{n} \in \mathcal{I}_{n}$, a based digraph $I_{n}^{*}$ will always have the base point 0 .

Definition 4.3. A path-map in a digraph $G$ is any digraph map $\phi: I_{n} \rightarrow G$, where $I_{n} \in \mathcal{I}_{n}$. A based path-map on a based digraph $G^{*}$ is a based digraph map $\phi: I_{n}^{*} \rightarrow G^{*}$, that is, a digraph map such that $\phi(0)=*$. A loop on $G^{*}$ is a based path-map $\phi: I_{n}^{*} \rightarrow G^{*}$ such that $\phi(n)=*$. 
Note that the image of a path-map is not necessary an allowed path of the digraph $G$.

Definition 4.4. A digraph map $h: I_{n} \rightarrow I_{m}$ is called shrinking if $h(0)=0$, $h(n)=m$, and $h(i) \leq h(j)$ whenever $i \leq j$ (that is, if $h$ as a function from $\{0, \ldots, n\}$ to $\{0, \ldots, m\}$ is monotone increasing $)$.

Any shrinking $h: I_{n} \rightarrow I_{m}$ is by definition a based digraph map. Moreover, $h$ is surjective and the preimage of any edge of $I_{m}$ consists of exactly one edge of $I_{n}$. Furthermore, we have necessarily $m \leq n$, and if $n=m$ then $h$ is a bijection.

Definition 4.5. Consider two based path-maps

$$
\phi: I_{n}^{*} \rightarrow G^{*} \text { and } \psi: I_{m}^{*} \rightarrow G^{*} .
$$

An one-step direct $C$-homotopy from $\phi$ to $\psi$ is given by a shrinking map $h: I_{n} \rightarrow I_{m}$ such that the map $F: V_{\mathrm{C}_{h}} \rightarrow V_{G}$ given by

$$
\left.F\right|_{I_{n}}=\phi \quad \text { and }\left.\quad F\right|_{I_{m}}=\psi
$$

is a digraph map from $\mathrm{C}_{h}$ to $G$. If the same is true with $\mathrm{C}_{h}$ replaced everywhere by $\mathrm{C}_{h}^{-}$then we refer to an one-step inverse $C$-homotopy.

Remark 4.6. The requirement that $F$ is a digraph map is equivalent to the condition

$$
\phi(i) \rightrightarrows \psi(h(i)) \text { for all } i \in I_{n}
$$

In turn, (4.2) implies that the digraph maps $\phi$ and $\psi \circ h$ (acting from $I_{n}$ to $G$ ) satisfy (3.2), which yields $\phi \simeq \psi \circ h$.

If $n=m$ then $h=\operatorname{id}_{I_{n}}$ and an one-step $C$-homotopy is a homotopy.

Example 4.7. An example of one-step direct $C$-homotopy is shown in Fig. 9.

Note that the images of the loops $\phi$ and $\psi$ on Fig. 9 are not homotopic as digraphs because they are cycles of different lengths 5 and 3 (see Example 3.13). Nevertheless, the loops $\phi$ and $\psi$ are $C$-homotopic.

Definition 4.8. For a based digraph $G^{*}$ define a path-digraph $P G$ as follows. The vertices of $P G$ are all the based path-maps in $G^{*}$, and the edges of 


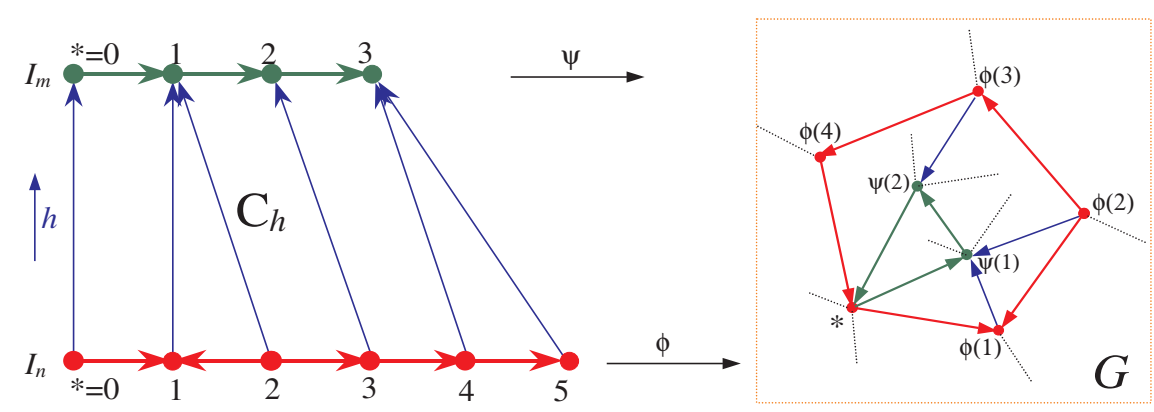

Figure 9: The loops $\phi: I_{5} \rightarrow G$ and and $\psi: I_{3} \rightarrow G$ are $C$-homotopic. Note that $\phi(0)=\phi(5)=*=\psi(0)=\psi(3)$.

$P G$ are defined by the following rule: $\phi \rightarrow \psi$ in $P G$ if $\phi \neq \psi$ and there is an one-step direct $C$-homotopy from $\phi$ to $\psi$ or an one-step inverse $C$-homotopy from $\psi$ to $\phi$.

Then define a based path-digraph $P G^{*}$ by choosing in $P G$ the base vertex $I_{0}^{*} \rightarrow G^{*}$, which will also be denoted by $*$. Define a based loop-digraph $L G^{*}$ as a sub-digraph of $P G^{*}$ whose set of vertices consists of all the loops of $G^{*}$.

Any map $f: G^{*} \rightarrow H^{*}$ induces a based map of path-digraphs

$$
\text { Pf : } P G^{*} \rightarrow P H^{*}, \quad(P f)(\psi)=f \circ \psi,
$$

where $\psi: I_{n}^{*} \rightarrow G^{*}$ is a based path-map. Hence, $P$ is a functor from the category $\mathcal{D}^{*}$ to itself. Similarly we have a map of based loop digraphs

$$
L f: L G^{*} \rightarrow L H^{*}, \quad(L f)(\psi)=f \circ \psi,
$$

where $\psi: I_{n}^{*} \rightarrow G^{*}$ is a loop. Hence, $L$ is a functor from the category $\mathcal{D}^{*}$ to itself.

Definition 4.9. We call two based path-maps $\phi, \psi \in P G$ C-homotopic and write $\phi \stackrel{C}{\simeq} \psi$ if there exists a finite sequence $\left\{\phi_{k}\right\}_{k=0}^{m}$ of based pathmaps in $P G$ such that $\phi_{0}=\phi, \phi_{m}=\psi$ and, for any $k=0, \ldots, m-1$, holds $\phi_{k} \rightarrow \phi_{k+1}$ or $\phi_{k+1} \rightarrow \phi_{k}$.

Obviously, the relation $\phi \stackrel{C}{\simeq} \psi$ holds if and only if $\phi$ and $\psi$ belong to the same connected component of the undirected graph of $P G$. In particular, the $C$-homotopy is an equivalence relation. 
Definition 4.10. Let $\pi_{1}\left(G^{*}\right)$ be a set of equivalence classes under $C$ homotopy of based loops of a digraph $G^{*}$. The $C$-homotopy class of a based loop $\phi$ will be denoted by $[\phi]$.

Note that $\pi_{1}\left(G^{*}\right)=\pi_{0}\left(L G^{*}\right)$ as follows directly from Definitions 4.8 and 4.10. Denote by $e$ the trivial loop $e: I_{0}^{*} \rightarrow G^{*}$. We say that a loop $\phi$ is $C$-contractible if $\phi \stackrel{C}{\simeq}$.

Example 4.11. A triangular loop is a loop $\phi: I_{3}^{*} \rightarrow G^{*}$ such that $I_{3}=$ $(0 \rightarrow 1 \rightarrow 2 \leftarrow 3)$.
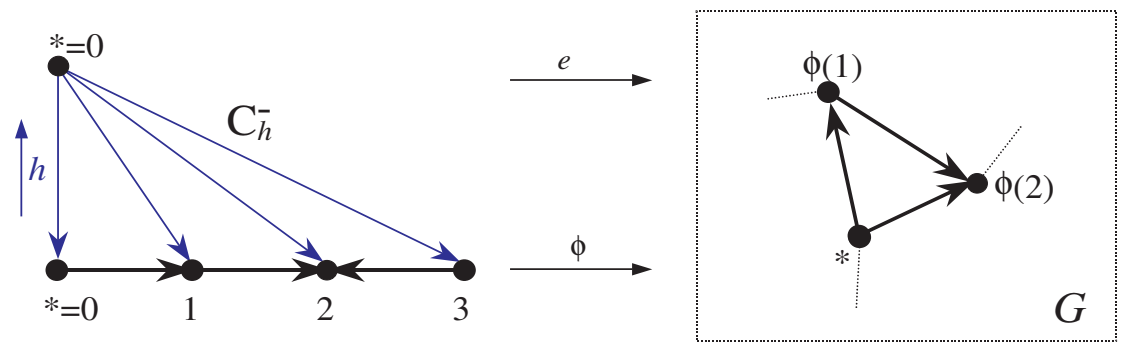

Figure 10: A triangular loop $\phi$ is $C$-contractible.

The triangular loop is $C$-contractible because the following shrinking map

$$
h: I_{3}^{*} \rightarrow I_{0}^{*}, \quad h(k)=0 \text { for all } k=0, \ldots, 3,
$$

provides an inverse one-step $C$-homotopy between $\phi$ and $e$ (see Fig. 10).

A square loop is a loop $\phi: I_{4}^{*} \rightarrow G$ such that $I_{4}=(0 \rightarrow 1 \rightarrow 2 \leftarrow 3 \leftarrow 4)$. The square loop can be $C$-contracted to $e$ in two steps as is shown on Fig. 11.

On the other hand, in the case $n \geq 5$, a loop $\phi: I_{n}^{*} \rightarrow G^{*}$ does not have to be $C$-contractible, which is the case, for example, if $\phi$ is the natural map $I_{n} \rightarrow S_{n}$.

\subsection{Local description of $C$-homotopy}

We prove here technical results which has a self-sustained meaning for practical work with $C$-homotopies.

Lemma 4.12. Let $a, b$ be two vertices in a digraph $G$ such that either $a=b$ or $a \rightarrow b \rightarrow a$. Then any path-map $\phi: I_{n} \rightarrow G$, such that $\phi(i)=a$, 

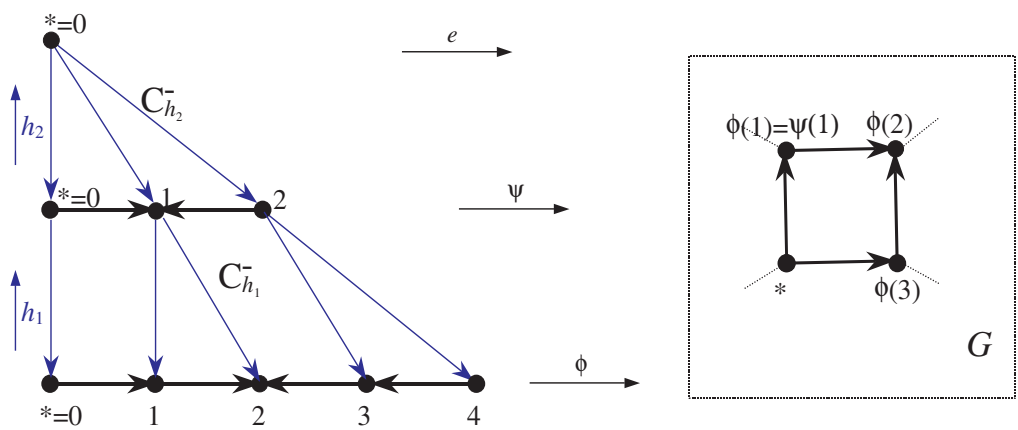

Figure 11: A square loop $\phi$ is $C$-contractible. Note that $\phi(0)=\phi(4)=$ $\psi(0)=\psi(2)=*$.

$\phi(i+1)=b$, and $i \rightarrow i+1$ in $I_{n}$, is $C$-homotopic to a path-map $\phi^{\prime}: I_{n}^{\prime} \rightarrow G$ where $I_{n}^{\prime}$ is obtained from $I_{n}$ by changing one edge $i \rightarrow i+1$ to $i+1 \rightarrow i$ and $\phi^{\prime}(j)=\phi(j)$ for all $j=0, \ldots, n$.

Proof. A $C$-homotopy between $\phi$ and $\phi^{\prime}$ is constructed in two one-step inverse $C$-homotopies as is shown on the following diagram:

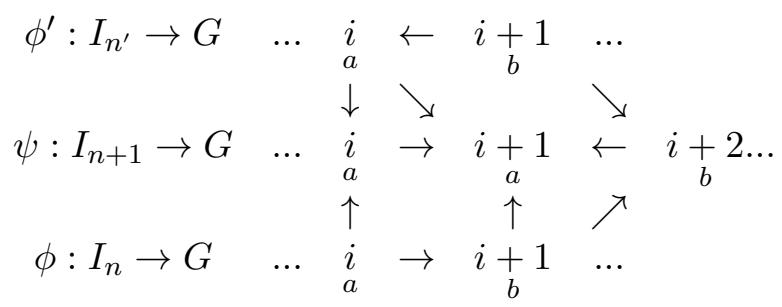

The subscript under each element of the line digraph indicates the value of the loop on this element.

Any path-map $\phi: I_{n} \rightarrow G$ defines a sequence $\theta_{\phi}=\left\{v_{i}\right\}_{i=0}^{n}$ of vertices of $G$ by $v_{i}=\phi(i)$. By definition of a path-map, we have for any $i=0, \ldots, n-1$ one of the following relations:

$$
v_{i}=v_{i+1}, \quad v_{i} \rightarrow v_{i+1}, \quad v_{i+1} \rightarrow v_{i}
$$

If $\phi$ is a based path-map, then we have $v_{0}=*$, if $\phi$ is a loop then $v_{0}=*=v_{n}$. We consider $\theta_{\phi}$ as a word over the alphabet $V_{G}$. 
Theorem 4.13. Two loops $\phi: I_{n}^{*} \rightarrow G^{*}$ and $\psi: I_{m}^{*} \rightarrow G^{*}$ are C-homotopic if and only if the word $\theta_{\psi}$ can be obtained from $\theta_{\phi}$ by a finite sequence of the following transformations (or inverses to them):

$(i) \ldots a b c \ldots \mapsto \ldots a c \ldots$ where $(a, b, c)$ is any permutation of a triple $\left(v, v^{\prime}, v^{\prime \prime}\right)$ of vertices forming a triangle in $G$, that is, such that $v \rightarrow v^{\prime}, v \rightarrow v^{\prime \prime}, v^{\prime} \rightarrow v^{\prime \prime}$ (and the dots "..." denote the unchanged parts of the words).

(ii) ...abc... $\mapsto \ldots a d c \ldots$ where $(a, b, c, d)$ is any cyclic permutation (or a cyclic permutation in the inverse order) of a quadruple $\left(v, v^{\prime}, v^{\prime \prime}, v^{\prime \prime \prime}\right)$ of vertices forming a square in $G$, that is, such that $v \rightarrow v^{\prime}, v \rightarrow v^{\prime \prime \prime}, v^{\prime} \rightarrow v^{\prime \prime}, v^{\prime \prime \prime} \rightarrow$ $v^{\prime \prime}$.

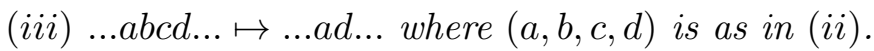

(iv) $\ldots a b a \ldots \rightarrow \ldots a \ldots$ if $a \rightarrow b$ or $b \rightarrow a$.

$(v) \ldots a a \ldots \mapsto \ldots a \ldots$

Proof. Let us first show that if $\theta_{\phi}=\theta_{\psi}$ then $\phi \stackrel{C}{\simeq} \psi$. If, for any edge $i \rightarrow i+1$ (or $i \leftarrow i+1$ ) in $I_{n}$ we have also $i \rightarrow i+1$ (resp. $i \leftarrow i+1$ ) in $I_{m}$ then $I_{n}=I_{m}$ and $\phi=\psi$ (although $n=m$, the line digraphs $I_{n}$ and $I_{m}$ could a priori be different elements of $\left.\mathcal{I}_{n}\right)$. Assume that, for some $i$, we have $i \rightarrow i+1$ in $I_{n}$ but $i \leftarrow i+1$ in $I_{m}$. Then, by Lemma 4.12, we can change the edge $i \rightarrow i+1$ in $I_{n}$ to $i \leftarrow i+1$ while staying in the same $C$-homotopy class of $\phi$. Arguing by induction, we obtain $\phi \stackrel{C}{\simeq} \psi$.

We write $\theta_{\phi} \sim \theta_{\psi}$ if $\theta_{\psi}$ can be obtained from $\theta_{\phi}$ by a finite sequence of transformations $(i)-(v)$ (or inverses to them). Let us show that $\theta_{\phi} \sim \theta_{\psi}$ implies that $\phi \stackrel{C}{\simeq} \psi$. For that we construct for each of the transformations $(i)-(v)$ a $C$-homotopy between $\phi$ and $\psi$. Note that in this part of the proof $\phi$ and $\psi$ can be arbitrary path-maps (not necessarily based).

(i) Assume that $a \rightarrow c$ (the case $c \rightarrow a$ is similar). Then either $b \rightarrow c$ or $a \rightarrow b$ (otherwise we would have got $a \rightarrow c \rightarrow b \rightarrow a$ which is excluded by a triangle hypothesis). The $C$-homotopies in the both cases are shown on the diagram:

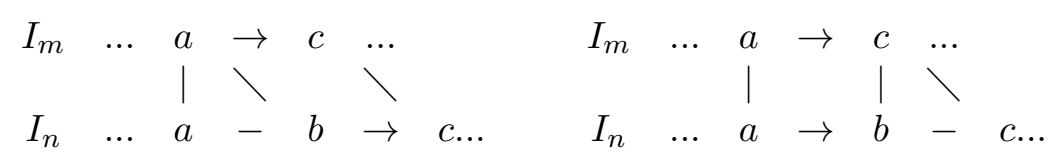

Each position here corresponds to a vertex in a cylinder $\mathrm{C}_{h}$ or $\mathrm{C}_{h}^{-}$(that is, in $I_{n}$ or $\left.I_{m}\right)$ and shows its image $(a, b$ or $c)$ under the map $\phi$ resp. $\psi$. The arrows and undirected segments shows the edges in the cylinder $\mathrm{C}_{h}$ or $\mathrm{C}_{h}^{-}$ (in particular, horizontal arrows and segments show the edges in $I_{n}$ and $I_{m}$ ). 
The undirected segments, such as $a-b$ and $c-b$, should be given directions matching those on the digraph $G$.

(ii) Assume as above $a \rightarrow d$ and $b \rightarrow c$. Then we have two-step $C$ homotopy as on the diagram:

$$
\begin{array}{ccccccccc}
I_{m} & \ldots & a & \rightarrow & d & - & c & \ldots & \\
& & \uparrow & \nwarrow & \nwarrow & & \nwarrow & \\
I_{n+1} & \ldots & a & - & a & \rightarrow & d & - & c \ldots \\
& & \mid & & \mid & & \mid & / & \\
I_{n} & \ldots & a & - & b & \rightarrow & c & \ldots &
\end{array}
$$

(iii) Assume $a \rightarrow d$. Then we have $b \rightarrow c$, and the $C$-homotopy is shown on the diagram:

$$
\begin{array}{llllllll}
I_{m} & \ldots & & \ldots & a & \rightarrow & d \\
& & \multicolumn{1}{l}{} & \mid & & \mid \\
I_{n} & \ldots & a & - & b & \rightarrow & c & -
\end{array}
$$

Note that if $a \rightarrow b$ then also $d \rightarrow c$, and if $b \rightarrow a$ then also $c \rightarrow d$.

(iv) Assuming $a \rightarrow b$ we obtain the following $C$-homotopy:

$$
\begin{array}{lllllll}
I_{m} & \ldots & & \ldots & a & \ldots & \\
& & & \swarrow & \downarrow & \searrow & \\
I_{n} & \ldots & a & \rightarrow & b & \leftarrow & a \ldots
\end{array}
$$

(v) Here is the required $C$-homotopy:

$$
\begin{array}{ccccccc}
I_{m} & \ldots & & \ldots & a & \ldots \\
& & & \nearrow & \uparrow & \\
I_{n} & \ldots & a & - & a & \ldots
\end{array}
$$

Before we go to the second half of the proof, observe that the transformation

$$
\ldots a b c \ldots \mapsto \ldots a c \ldots
$$

of words is possible not only in the case when $a, b, c$ come from a triangle as in $(i)$ but also when $a, b, c$ form a degenerate triangle, that is, when there are identical vertices among $a, b, c$ while distinct vertices among $a, b, c$ are 
connected by an edge. Indeed, in the case $a=b$ we have by $(v)$

$$
a b c=a a c \sim a c,
$$

in the case $a=c$ we have by $(i v)$ and $(v)$

$$
a b c=a b a \sim a \sim a c,
$$

and in the case $b=c$ by $(v)$

$$
a b c=a c c \sim a c .
$$

Now let us prove that $\phi \stackrel{C}{\simeq} \psi$ implies $\theta_{\phi} \sim \theta_{\psi}$. It suffices to assume that there exists an one-step direct $C$-homotopy from $\phi$ to $\psi$ given by a shrinking $\operatorname{map} h: I_{n}^{*} \rightarrow I_{m}^{*}$. Set

$$
\theta_{\phi}=a_{0} a_{1} \ldots a_{n} \text { and } \theta_{\psi}=b_{0} b_{1} \ldots b_{m}
$$

where $a_{i}, b_{j} \in V_{G}$ and $a_{0}=b_{0}=a_{n}=b_{m}=*$. For any $i=0, \ldots, n$ set $j=$ $h(i)$ and consider two words

$$
A_{i}=a_{0} a_{1} \ldots a_{i} b_{j} \quad \text { and } \quad B_{i}=b_{0} b_{1} \ldots b_{j}
$$

We will prove by induction in $i$ that $A_{i} \sim B_{i}$ for all $i=0, \ldots, n$. If this is already known, then for $i=n$ we have $j=m$ and

$$
a_{0} a_{1} \ldots a_{n} b_{m} \sim b_{0} b_{1} \ldots b_{m}
$$

Since $a_{n} b_{m}=* * \sim *=a_{n}$, it follows that $\theta_{\phi} \sim \theta_{\psi}$.

Now let us prove that $A_{i} \sim B_{i}$ for all $i=0, \ldots, n$. For $i=0$ we have $A_{0}=a_{0} b_{0}=* * \sim *=b_{0}=B_{0}$. Assuming that $A_{i} \sim B_{i}$, let us prove that $A_{i+1} \sim B_{i+1}$. Let us consider a structure of the cylinder $\mathrm{C}_{h}$ over the edge between $i$ and $i+1$ in $I_{n}$. Set $h(i)=j, a=a_{i}, a^{\prime}=a_{i+1}, b=b_{j}, b^{\prime}=b_{j+1}$. There are only the following two cases:

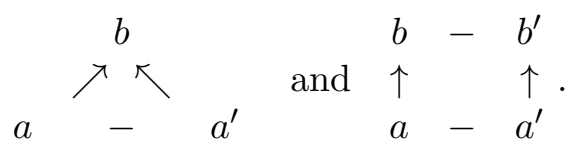

Note that each arrow on $\mathrm{C}_{h}$ transforms either to an arrow between the vertices of $G$ or to the identity of the vertices. 
Consider first the case of the left diagram in (4.5). In this case $b^{\prime}=b$ and we obtain by (4.4) and by the induction hypothesis that

$$
A_{i+1}=a_{0} a_{1} \ldots a_{i-1} a a^{\prime} b \sim a_{0} a_{1} \ldots a_{i-1} a b=A_{i} \sim B_{i}=B_{i+1} .
$$

Consider now the case of the right diagram in (4.5) and prove that in this case

$$
a a^{\prime} b^{\prime} \sim a b b^{\prime}
$$

If (4.6) is already known, then we obtain

$$
A_{i+1}=a_{0} a_{1} \ldots a_{i-1} a a^{\prime} b^{\prime} \sim a_{0} a_{1} \ldots a_{i-1} a b b^{\prime}=A_{i} b^{\prime} \sim B_{i} b^{\prime}=B_{i+1}
$$

which concludes the induction step in this case.

In order to prove (4.6) observe first that if all the vertices $a, a^{\prime}, b, b^{\prime}$ are distinct, then they form a square and (4.6) follows by transformation $(i i)$. In the case $a^{\prime}=b(4.6)$ is an equality, and in the case $a=b^{\prime}$ the relation (4.6) follows by transformation $(i v)$ :

$$
a a^{\prime} b^{\prime} \sim a=b^{\prime} \sim a b b^{\prime}
$$

In the case $a=b$ the triple $a, a^{\prime}, b^{\prime}$ is a triangle or a degenerate triangle, and we obtained from (4.4) and $(v)$

$$
a a^{\prime} b^{\prime} \sim a b^{\prime} \sim a a b^{\prime}=a b b^{\prime}
$$

and the case $a^{\prime}=b^{\prime}$ is similar. Finally, if $a=a^{\prime}$ then similarly by $(v)$ and (4.4) we obtain

$$
a a^{\prime} b^{\prime}=a a b^{\prime} \sim a b^{\prime} \sim a b b^{\prime}
$$

and the case $b=b^{\prime}$ is similar.

Remark 4.14. Note that the transformation (iii) was not used in the second half of the proof, so $(i i i)$ is logically not necessary in the statement of Theorem 4.13. Note also that $($ iii) can be obtained as composition of $(i i)$ and $(i v)$ as follows:

$$
a b c d \sim a d c d \sim a d
$$

However, in applications it is still convenient to be able to use $(i i i)$. 
Example 4.15. A triangular loop on Fig. 10 is contractible because if $a, b, c$ are vertices of a triangle then

$$
a b c a \sim a c a \sim a
$$

A square loop on Fig. 11 is contractible because if $a, b, c, d$ are vertices of a square then

$$
a b c d a \sim a d a \sim a
$$

Consider the loops $\phi$ and $\psi$ on Fig. 9, that are known to be $C$-homotopic. It is shown on Fig. 12 how to transform $\theta_{\phi}$ to $\theta_{\psi}$ using transformations of Theorem 4.13.
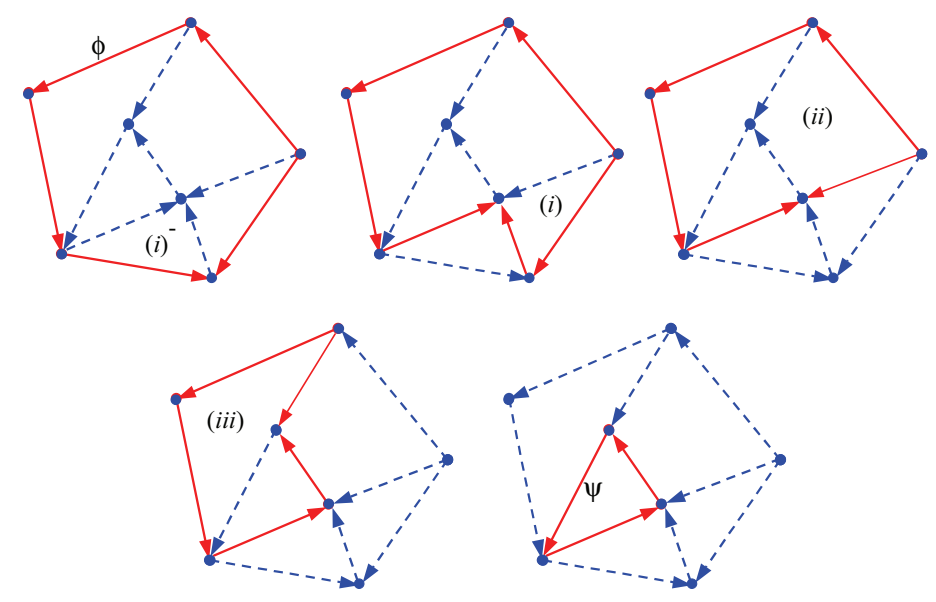

Figure 12: Transforming a 5-cycle $\theta_{\phi}$ to a 3 -cycle $\theta_{\psi}$ using successively $(i)^{-}$ (the inverse of $(i)),(i),(i i)$ and $(i i i)$.

\subsection{Group structure in $\pi_{1}$}

For any $I_{n} \in \mathcal{I}_{n}$ define a line digraph $\hat{I}_{n} \in \mathcal{I}_{n}$ as follows:

$$
i \rightarrow j \text { in } \hat{I}_{n} \Leftrightarrow(n-i) \rightarrow(n-j) \text { in } I_{n}
$$

For any two line digraphs $I_{n}$ and $I_{m}$, define the line digraph $I_{n+m}=I_{n} \vee$ $I_{m} \in \mathcal{I}_{n+m}$ that is obtained from $I_{n}$ and $I_{m}$ by identification of the vertices $n \in I_{n}$ and $0 \in I_{m}$. 
Definition 4.16. ( $i$ ) For a path-map $\phi: I_{n} \rightarrow G$ define the inverse path$\operatorname{map} \hat{\phi}: \hat{I}_{n} \rightarrow G$ by $\hat{\phi}(i)=\phi(n-i)$.

(ii) For two path-maps $\phi: I_{n} \rightarrow G$ and $\psi: I_{m} \rightarrow G$ with $\phi(n)=\psi(0)$ define the concatenation path-map $\phi \vee \psi: I_{n+m} \rightarrow G$ by

$$
\phi \vee \psi(i)= \begin{cases}\phi(i), & 0 \leq i \leq n \\ \psi(i-n), & n \leq i \leq n+m .\end{cases}
$$

The operation $\phi \mapsto \hat{\phi}$ is evidently an involution on the set of path-maps. Clearly, if $\phi$ is a loop in $G^{*}$ then $\hat{\phi}$ is also a loop, and the concatenation of two loops is also a loop. Let us define a product in $\pi_{1}\left(G^{*}\right)$ as follows.

Definition 4.17. For any two loops

$$
\phi: I_{n}^{*} \rightarrow G^{*} \text { and } \psi: I_{m}^{*} \rightarrow G^{*}
$$

define the product of $[\phi]$ and $[\psi]$ by

$$
[\phi] \cdot[\psi]=[\phi \vee \psi]
$$

where $\phi \vee \psi: I_{n+m}^{*} \rightarrow G^{*}$ is the concatenation of $\phi$ and $\psi$.

Lemma 4.18. The product in $\pi_{1}\left(G^{*}\right)$ is well defined.

Proof. Let $\phi, \phi^{\prime}, \psi, \psi^{\prime}$ be loops of $G^{*}$ and let

$$
\phi \stackrel{C}{\simeq} \phi^{\prime}, \quad \psi \stackrel{C}{\simeq} \psi^{\prime}
$$

We must prove that

$$
\phi \vee \psi \stackrel{C}{\simeq} \phi^{\prime} \vee \psi^{\prime}
$$

It suffices to consider only the case when the both $C$-homotopies in (4.8) are one-step $C$-homotopies. Then we have

$$
\phi \vee \psi \stackrel{C}{\simeq} \phi^{\prime} \vee \psi
$$

because one-step $C$-homotopy between $\phi$ and $\phi^{\prime}$ easily extends to that between $\phi \vee \psi$ and $\phi^{\prime} \vee \psi$. In the same way we obtain

$$
\phi^{\prime} \vee \psi \stackrel{C}{\simeq} \phi^{\prime} \vee \psi^{\prime}
$$

whence (4.9) follows. 
Lemma 4.19. For any loop $\phi: I_{n}^{*} \rightarrow G^{*}$ we have $\phi \vee \hat{\phi} \stackrel{C}{\simeq}$ e where $\hat{\phi}$ is the inverse loop for the loop $\phi$ and

$$
e: I_{0}^{*} \rightarrow G^{*}
$$

is the trivial loop.

Proof. Let $\theta_{\phi}=v_{0} \ldots v_{n}$. Then $\theta_{\hat{\phi}}=v_{n} \ldots v_{0}$ and

$$
\theta_{\phi \vee \hat{\phi}}=v_{0} \ldots v_{n-1} v_{n} v_{n-1} \ldots v_{0}
$$

Using successively the transformations $a b a \mapsto a$ and $a a \mapsto a$ of Theorem 4.13, we obtain that $\theta_{\phi \vee \hat{\phi}} \sim *$ whence $\phi \vee \hat{\phi} \stackrel{C}{\simeq} e$ follows.

Theorem 4.20. Let $G, H$ be digraphs.

(i) The set $\pi_{1}\left(G^{*}\right)$ with the product (4.7) and neutral element [e] from (4.10) is a group. It will be referred to as the fundamental group of a digraph $G^{*}$.

(ii) A based digraph map $f: G^{*} \rightarrow H^{*}$ induces a group homomorphism

$$
\pi_{1}(f): \pi_{1}\left(G^{*}\right) \rightarrow \pi_{1}\left(H^{*}\right), \quad\left(\pi_{1}(f)\right)[\phi]=[f \circ \phi]
$$

which depends only on homotopy class of $f$. Hence, we obtain a functor from the category of digraphs $\mathcal{D}^{*}$ to the category of groups.

(iii) Let $\gamma: I_{k}^{*} \rightarrow G^{*}$ be a based path-map with $\gamma(k)=v$. Then $\gamma$ induces an isomorphism of fundamental groups

$$
\gamma_{\sharp}: \pi_{1}\left(G^{*}\right) \rightarrow \pi_{1}\left(G^{v}\right),
$$

which depends only on C-homotopy class of the path-map $\gamma$.

Proof. ( $i$ ) This follows from Lemmas 4.18 and 4.19, since the product in $\pi_{1}\left(G^{*}\right)$ satisfies the associative law, the class $[e] \in \pi_{1}\left(G^{*}\right)$ satisfies the definition of a neutral element, and $[\hat{\phi}]$ is the inverse of $[\phi]$ for any $[\phi] \in \pi_{1}\left(G^{*}\right)$.

(ii) Let $\phi$ and $\psi$ be $C$-homotopic loops in $G^{*}$. It follows from Definition 4.5 and (4.2) that $f \circ \phi \stackrel{C}{\simeq} f \circ \psi$ and, hence, the map $\pi_{1}(f)$ is well defined. 
The map $\pi_{1}(f)$ is a homomorphism because $\pi_{1}([e])=[e]$ and, for any two loops $\phi, \phi^{\prime}$ in $G^{*}$,

$$
f \circ\left(\phi \vee \phi^{\prime}\right)=(f \circ \phi) \vee\left(f \circ \phi^{\prime}\right) .
$$

If $f$ and $g$ two homotopic based maps from $G^{*}$ to $H^{*}$ then $f \circ \phi \simeq g \circ \phi$ and hence $f \circ \phi \stackrel{C}{\simeq} g \circ \phi$, which finishes the proof.

(iii) For any loop $\phi$ in $G^{*}$, define a based loop $\gamma_{\sharp}(\phi)$ in $G^{v}$ by

$$
\gamma_{\sharp}(\phi)=\hat{\gamma} \vee \phi \vee \gamma: I_{k+n+k} \rightarrow G,
$$

where $\hat{\gamma}$ is the inverse path-map of $\gamma$ as in Definition 4.16. Similarly to the proof of $(i i)$ and using Lemma 4.19, one shows that $\gamma_{\sharp}: \pi_{1}(G, *) \rightarrow \pi_{1}(G, v)$ is a group homomorphism. Since $\hat{\gamma}_{\sharp}$ is obviously the inverse map of $\gamma_{\sharp}$, it follows that $\gamma_{\sharp}$ is an isomorphism.

If $\gamma_{1}$ and $\gamma_{2}$ are two $C$-homotopic path-maps connecting vertices $*$ and $v$ then $\hat{\gamma}_{1} \vee \phi \vee \gamma_{1}$ and $\hat{\gamma}_{2} \vee \phi \vee \gamma_{2}$ are $C$-homotopic (cf. the proof of Lemma 4.18). Hence, $\gamma_{\sharp}$ depends only on $C$-homotopy class of the map $\gamma$.

Lemma 4.21. Let $f: G^{*} \rightarrow H^{a}$ and $g: G^{*} \rightarrow H^{b}$ be two based digraphs maps. If $f \simeq g: G \rightarrow H$ then there exists a based path-map $\gamma: I_{k}^{*} \rightarrow H^{a}$ with $\gamma(k)=b$ such that, for any loop $\phi: I_{n}^{*} \rightarrow G^{*}$, we have

$$
\gamma_{\sharp}(f \circ \phi) \stackrel{C}{\simeq} g \circ \phi .
$$

Consequently, the following diagram is commutative:

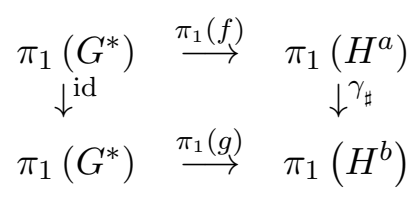

Proof. Note that $f \circ \phi$ is a loop in $H^{a}$ and $g \circ \phi$ is a loop in $H^{b}$. It suffices to prove the statement in the case when $f$ and $g$ are related by an one-step homotopy, that is, $f(x) \rightrightarrows g(x)$ for all $x \in V_{G}$. In particular, we have $a \rightrightarrows b$.

Consider the path-map $\gamma: I \rightarrow H$ given by $\gamma(0)=a$ and $\gamma(1)=b$. Then the loop $\gamma_{\sharp}(f \circ \phi): \hat{I} \vee I_{n} \vee I \rightarrow H^{b}$ is defined by

$$
\gamma_{\sharp}(f \circ \phi)=\hat{\gamma} \vee(f \circ \phi) \vee \gamma .
$$

Define shrinking $h: \hat{I} \vee I_{n} \vee I \rightarrow I_{n}$ as follows: $h$ on $I_{n}$ is identical, and the endpoints of $\hat{I} \vee I_{n} \vee I$ are mapped by $h$ to the corresponding endpoints of 
$I_{n}:$

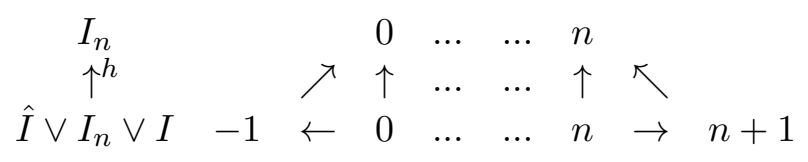

where we enumerate the vertices of $\hat{I} \vee I_{n} \vee I$ as $\{-1,0, \ldots, n+1\}$.

Then we have, for $0 \leq i \leq n$,

$$
\gamma_{\sharp}(f \circ \phi)(i)=f(\varphi(i)) \rightrightarrows g(\phi(i))=(g \circ \varphi)(h(i)),
$$

for $i=-1$

$$
\gamma_{\sharp}(f \circ \phi)(-1)=b=g(\varphi(0))=(g \circ \varphi)(h(-1)),
$$

and for $i=n+1$

$$
\gamma_{\sharp}(f \circ \phi)(n+1)=b=g(\varphi(n))=(g \circ \varphi)(h(n+1)) .
$$

Hence, for all $i$,

$$
\gamma_{\sharp}(f \circ \phi)(i) \rightrightarrows(g \circ \varphi)(h(i)),
$$

which implies (4.11) by (4.2).

Theorem 4.22. Let $G, H$ be two connected digraphs. If $G \simeq H$ then the fundamental groups $\pi_{1}\left(G^{*}\right)$ and $\pi_{1}\left(H^{*}\right)$ are isomorphic (for any choice of the based vertices).

Proof. Let $f: G \rightarrow H$ and $g: H \rightarrow G$ be homotopy inverses maps (cf. 3.4). Applying Lemma 4.21 to $f \circ g \simeq \operatorname{id}_{G}$ and to $g \circ f \simeq \mathrm{id}_{H}$, we obtain the result by a standard argument (cf. [12, Ch.1, Thm 8]).

\subsection{Relation between $H_{1}$ and $\pi_{1}$}

One of our main results is the following theorem.

Theorem 4.23. For any based connected digraph $G^{*}$ we have an isomorphism

$$
\pi_{1}\left(G^{*}\right) /\left[\pi_{1}\left(G^{*}\right), \pi_{1}\left(G^{*}\right)\right] \cong H_{1}(G, \mathbb{Z})
$$

where $\left[\pi_{1}\left(G^{*}\right), \pi_{1}\left(G^{*}\right)\right]$ is a commutator subgroup. 
Proof. The proof is similar to that in the classical algebraic topology $[8$, p.166]. For any based loop $\phi: I_{n}^{*} \rightarrow G^{*}$ of a digraph $G^{*}$, define a 1-path $\chi(\phi)$ on $G$ as follows: $\chi(\phi)=0$ for $n=0,1,2$, and for $n \geq 3$

$$
\chi(\phi)=\sum_{\{i: i \rightarrow i+1\}} e_{\phi(i) \phi(i+1)}-\sum_{\{i: i+1 \rightarrow i\}} e_{\phi(i+1) \phi(i)},
$$

where the summation index $i$ runs from 0 to $n-1$. It is easy to see that the 1-path $\chi(\phi)$ is allowed and closed and, hence, determines a homology class $[\chi(\phi)] \in H_{1}(G, \mathbb{Z})$. Let us first prove that, for any two based loops $\phi: I_{n}^{*} \rightarrow G^{*}$ and $\psi: I_{m}^{*} \rightarrow G^{*}$,

$$
\phi \stackrel{C}{\simeq} \psi \Rightarrow[\chi(\phi)]=[\chi(\psi)]
$$

Note that any based loop with $n \leq 2$ is $C$-homotopic to trivial. For $n \geq 3$, it is sufficiently to check (4.13) assuming that $\phi \stackrel{C}{\simeq} \psi$ is given by an one-step direct $C$-homotopy with a shrinking map $h: I_{n}^{*} \rightarrow I_{m}^{*}$. Set

$$
\phi^{\prime}:=\psi \circ h: I_{n}^{*} \rightarrow G^{*}
$$

and observe that by $(4.12) \chi\left(\phi^{\prime}\right)=\chi(\psi)$. It remains to show that $[\chi(\phi)]=$ $\left[\chi\left(\phi^{\prime}\right)\right]$.

By Remark 4.6 the digraph maps $\phi$ and $\phi^{\prime}$, acting from $I_{n}$ to $G$, are homotopic. Denote by $S_{n}$ the digraph that is obtained from $I_{n}$ by identification of the vertices 0 and $n$ (that is, $S_{n}$ is a cycle digraph from Example 2.8). Then $\varphi$ and $\phi^{\prime}$ can be regarded as digraph maps from $S_{n}$ to $G$, and they are again homotopic as such.

Consider the standard homology class $[\varpi] \in H_{1}\left(S_{n}\right)$ given by (2.8). Comparing (2.8) and (4.12), we see that

$$
\phi_{*}(\varpi)=\chi(\varphi) \text { and } \phi_{*}^{\prime}(\varpi)=\chi\left(\phi^{\prime}\right) .
$$

On the other hand, by Theorem 3.3 we have $\left[\phi_{*}(\varpi)\right]=\left[\phi_{*}^{\prime}(\varpi)\right]$, which finishes the proof of (4.13).

Hence, $\chi$ determines a map

$$
\chi_{*}: \pi_{1}\left(G^{*}\right) \rightarrow H_{1}(G, \mathbb{Z}), \quad \chi_{*}[\phi]=[\chi(\phi)] .
$$


The map $\chi_{*}$ is a group homomorphism because, for based loops $\phi, \psi$ and the neutral element $[e] \in \pi_{1}\left(G^{*}\right)$, we have $\chi_{*}([e])=0$ and

$$
\begin{aligned}
\chi_{*}([\phi] \cdot[\psi]) & =\chi_{*}([\phi \vee \psi])=[\chi(\phi \vee \psi)] \\
& =[\chi(\phi)+\chi(\psi)]=[\chi(\phi)]+[\chi(\psi)]=\chi_{*}([\phi])+\chi_{*}([\psi]) .
\end{aligned}
$$

Since the group $H_{1}(G, \mathbb{Z})$ is abelian, it follows that

$$
\left[\pi_{1}\left(G^{*}\right), \pi_{1}\left(G^{*}\right)\right] \subset \operatorname{Ker} \chi_{*}
$$

Now let us prove that $\chi_{*}$ is an epimorphism. Define a standard loop on $G$ as a finite sequence $v=\left\{v_{k}\right\}_{k=0}^{n}$ of vertices of $G$ such that $v_{0}=v_{n}$ and, for any $k=0, \ldots, n-1$, either $v_{k} \rightarrow v_{k+1}$ or $v_{k+1} \rightarrow v_{k}$. For a standard loop $v$ define an 1-path

$$
\varpi_{v}=\sum_{\left\{k: v_{k} \rightarrow v_{k+1}\right\}} e_{v_{k} v_{k+1}}-\sum_{\left\{k: v_{k+1} \rightarrow v_{k}\right\}} e_{v_{k} v_{k+1}}
$$

and observe that $\varpi_{v}$ is allowed and closed. The 1-paths of the form (4.14) will be referred to as standard paths. Consider an arbitrary closed 1-path

$$
w=\sum_{k} n_{k} e_{i_{k} j_{k}} \in \Omega_{1}(G, \mathbb{Z}) .
$$

Since $\partial w=0$ and $\partial e_{i j}=e_{j}-e_{i}$, the path $w$ can be represented as a finite sum of standard paths. Hence, in order to prove that $\chi_{*}$ is an epimorphism, it suffices to show that any standard 1-path $\varpi_{v}$ is in the image of $\chi$. Note that the standard loop $v$ determines naturally a based loop $\phi: I_{n}^{*} \rightarrow G^{v_{0}}$ by $\phi(i)=v_{i}$. Since the digraph $G$ is connected, there exists a based path $f: I_{s}^{*} \rightarrow G^{*}$ with $f(s)=v_{0}$. Thus we obtain a based loop

$$
f \vee \phi \vee \hat{f}: I_{2 s+n}^{*} \rightarrow G^{*}
$$

It follows directly from our construction, that $\chi(f \vee \phi \vee \hat{f})=\varpi_{v}$, and hence $\chi_{*}$ is an epimorphism.

We are left to prove that

$$
\operatorname{Ker} \chi_{*} \subset\left[\pi_{1}\left(G^{*}\right), \pi_{1}\left(G^{*}\right)\right]
$$

For that we need to prove that, for any loop $\phi: I_{n}^{*} \rightarrow G^{*}$, if $\chi_{*}([\phi])=0 \in$ $H_{1}(G, \mathbb{Z})$, then $[\phi]$ lies in the commutator $\left[\pi_{1}\left(G^{*}\right), \pi_{1}\left(G^{*}\right)\right]$. In the case $n \leq 2$ 
any loop $\phi$ is $C$-homotopic to the trivial loop. Assuming in the sequel $n \geq 3$, we use the word $\theta_{\phi}=v_{0} v_{1} \ldots v_{n}$ where $v_{i}=\phi(i)$.

Consider first the case, when $\chi(\phi)=0 \in \Omega_{1}(G)$. Since the digraph $G$ is connected, for any vertex $v_{i}$ there exists a based path-map $\psi_{i}: I_{p_{i}}^{*} \rightarrow$ $G^{*}$ with $\psi_{i}\left(p_{i}\right)=v_{i}$. If $v_{i}=v_{j}$ for some $i, j$ then we make sure to choose $\psi_{i}$ and $\psi_{j}$ identical. For $i=0$ and $i=n$ choose $\psi_{i}$ to be trivial path-map $e: I_{0}^{*} \rightarrow G^{*}$. For any $i=0, \ldots, n-1$ define path-map $\phi_{i}: I^{ \pm} \rightarrow G$ by the conditions $\phi_{i}(0)=v_{i}, \phi_{i}(1)=v_{i+1}$ and consider the following loop

$\gamma=\psi_{0} \vee \phi_{0} \vee \hat{\psi}_{1} \vee \psi_{1} \vee \phi_{1} \vee \hat{\psi}_{2} \vee \psi_{2} \vee \phi_{2} \vee \cdots \vee \hat{\psi}_{n-1} \vee \psi_{n-1} \vee \phi_{n-1} \vee \psi_{n}$

(see Fig. 13).

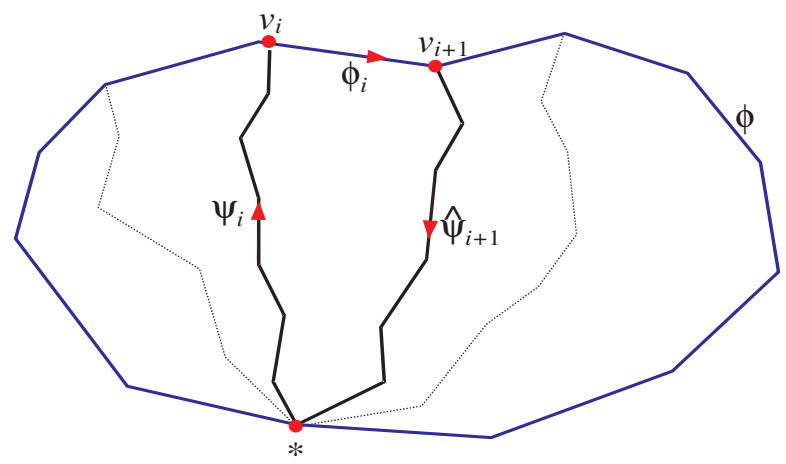

Figure 13: Loop $\psi_{i} \vee \phi_{i} \vee \hat{\psi}_{i+1}$

Using transformation (iv) of Theorem 4.13 (similarly to the proof of Lemma 4.19), we obtain that

$$
\gamma \stackrel{C}{\simeq} \phi_{0} \vee \phi_{1} \vee \ldots \vee \phi_{n-1}=\phi
$$

On the other hand, it follows from (4.15) that

$$
[\gamma]=\prod_{i=0}^{n-1}\left[\psi_{i} \vee \phi_{i} \vee \hat{\psi}_{i+1}\right]
$$

Consider for some $i=0, \ldots, n-1$, such that $i \rightarrow i+1$, the vertices $a=v_{i}$ and $b=v_{i+1}$. If $a=b$ then the loop $\psi_{i} \vee \phi_{i} \vee \hat{\psi}_{i+1}$ is $C$-homotopic to $e$. Assume $a \neq b$, so that $a \rightarrow b$. Then the term $e_{a b}$ is present in the right hand side 
of the identity (4.12) defining $\chi(\phi)$. Due to $\chi(\phi)=0$, the term $e_{a b}$ should cancel out with $-e_{a b}$ in the right hand side of (4.12). Therefore, there exists $j=0, \ldots, n-1$ such that $j+1 \rightarrow j, v_{j+1}=a$ and $v_{j}=b$. It follows that

$$
\psi_{j} \vee \phi_{j} \vee \hat{\psi}_{j+1}=\psi_{i+1} \vee \hat{\phi}_{i} \vee \hat{\psi}_{i}
$$

and that the loops

$$
\left[\psi_{i} \vee \phi_{i} \vee \hat{\psi}_{i+1}\right] \text { and }\left[\psi_{j} \vee \phi_{j} \vee \hat{\psi}_{j+1}\right]
$$

are mutually inverse. Therefore, $[\gamma]$ is a product of pairs of mutually inverse loops, which implies that $[\gamma]=[\phi]$ lies in the commutator of $\pi_{1}$.

Now consider the general case, when $\chi(\phi) \in \Omega_{1}(G)$ is exact, that is, $\chi(\phi)=\partial \omega$ for some $\omega \in \Omega_{2}(G)$. Recall that by Proposition 2.9 any 2-path $\omega \in \Omega_{2}$ can be represented in the form

$$
\omega=\sum_{j=1}^{N} \kappa_{j} \sigma_{j}
$$

where $N \in \mathbb{N}, \kappa_{l}= \pm 1$ and $\sigma_{l}$ is one of the following 2-paths: a double edge, a triangle, a square. Further proof goes by induction in $N$. In the case $N=0$ we have $\omega=0$ which was already considered above.

In the case $N \geq 1$ choose an arbitrary index $i=0, \ldots, n-1$ such that the vertices $a=\phi(i)$ and $b=\phi(i+1)$ are distinct. Assume for certainty that $i \rightarrow i+1$ and, hence, $a \rightarrow b$ (the case $i+1 \rightarrow i$ can be handled similarly). Then $e_{a b}$ enters $\chi(\phi)$ with the coefficient 1 . Since

$$
\chi(\phi)=\partial \omega=\sum_{j=1}^{N} \kappa_{j} \partial \sigma_{j},
$$

there exists $\sigma_{l}$ such that $\partial \sigma_{l}$ contains a term $\kappa_{l} e_{a b}$. Fix this $l$ and define a new loop $\phi^{\prime}$ as follows.

If $\sigma_{l}$ is a double edge $a, b, a$, then consider a loop $\phi^{\prime}$ that is obtained from $\phi: I_{n}^{*} \rightarrow G^{*}$ by changing one edge $i \rightarrow i+1$ in $I_{n}$ to $i \rightarrow i+1$. Then by Lemma 4.12 we have $\phi^{\prime} \stackrel{C}{\simeq} \phi$.

Let $\sigma_{l}$ be a triangle with the vertices $a, b, c$. Noticing that

$$
\theta_{\phi}=\ldots a b \ldots
$$


consider a loop $\phi^{\prime}$ such that

$$
\theta_{\phi^{\prime}}=\ldots a c b \ldots
$$

(see Fig. 14).

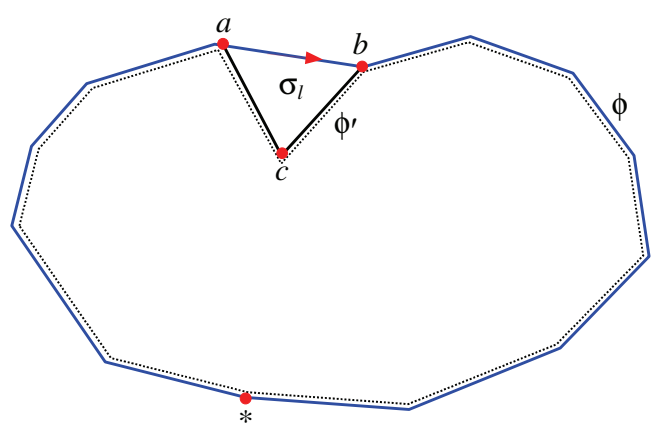

Figure 14: Loops $\phi$ and $\phi^{\prime}$ in the case when $\sigma_{l}$ is a triangle.

If $\sigma_{l}$ is a square with the vertices $a, b, c, d$, then we define a loop $\phi^{\prime}$ so that

$$
\theta_{\phi^{\prime}}=\ldots a d c b
$$

By Theorem 4.13, we have in the both cases $\phi^{\prime} \stackrel{C}{\simeq} \phi$ and, hence, $\left[\phi^{\prime}\right]=[\phi]$.

By construction, $\chi\left(\phi^{\prime}\right)$ contains no longer the term $e_{a b}$. On the other hand, we will prove below that, for some $\kappa= \pm 1$,

$$
\chi\left(\phi^{\prime}\right)=\chi(\phi)-\kappa \partial \sigma_{l}
$$

Comparing the coefficients in front of $e_{a b}$ in the both parts of (4.17), we obtain the identity $0=1-\kappa \kappa_{l}$ whence $\kappa=\kappa_{l}$. It follows from (4.17) with $\kappa=\kappa_{l}$ that

$$
\chi\left(\phi^{\prime}\right)=\chi(\phi)-\partial\left(\kappa_{l} \sigma_{l}\right)=\partial \omega-\partial\left(\kappa_{l} \sigma_{l}\right)=\partial \omega^{\prime},
$$

where

$$
\omega^{\prime}=\sum_{j \neq l} c_{j} \sigma_{j} .
$$

By the inductive hypothesis we conclude that $\left[\phi^{\prime}\right]$ lies in the commutator $\left[\pi_{1}\left(G^{*}\right), \pi_{1}\left(G^{*}\right)\right]$, whence the same for $[\phi]$ follows. 
We are left to prove the identity (4.17). If $\sigma_{l}$ is a double edge $a, b, a$ then

$$
\chi\left(\phi^{\prime}\right)-\chi(\phi)=-e_{b a}-e_{a b}=-\partial e_{a b a}=-\partial \sigma_{l}
$$

If $\sigma_{l}$ is a triangle

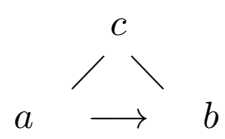

then we obtain a cycle digraph $S_{3}$ with the vertices $a, b, c$, and if $\sigma_{l}$ is a square

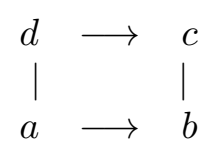

then we obtain a cycle digraph $S_{4}$ with the vertices $a, b, c, d$. Let $\varpi$ be the standard 1-path on $S_{3}$ in the first case and that on $S_{4}$ in the second case (see $(2.8))$. Then it is easy to see that

$$
\chi(\phi)-\chi\left(\phi^{\prime}\right)=\varpi
$$

and (4.17) follows from the observation that $\partial \sigma_{l}= \pm \varpi$ (cf. Example 2.8).

\subsection{Higher homotopy groups}

Recall that, for any based digraph $G^{*}$, a based loop-digraph $L G^{*}$ was defined in Definition 4.8, and, for a digraph map $f: G^{*} \rightarrow H^{*}$, we defined a digraph map $L f: L G^{*} \rightarrow L H^{*}$ by (4.3).

Definition 4.24. For any digraph $G^{*}$ let $L^{n} G=L^{n} G^{*}, n=0,1,2,3, \ldots$ be based digraphs defined inductively as

$$
L^{0} G^{*}=G^{*}, \quad L^{1} G^{*}=L G^{*}, \quad \text { and, for } n \geq 2, \quad L^{n} G^{*} \stackrel{\text { def }}{=} L\left(L^{n-1} G^{*}\right)
$$

where the base point in $L G^{*}$ is the based map $I_{0}^{*} \rightarrow G^{*}$ which we also denote by $*$.

For $n \geq 2$, define homotopy group $\pi_{n}\left(G^{*}\right)$ of the digraph $G^{*}$ inductively by

$$
\pi_{n}\left(G^{*}\right)=\pi_{n-1}\left(L G^{*}\right) .
$$


Theorem 4.25. Let $G^{*}, H^{*}$ be two based digraphs. If $f$ and $g$ are homotopic digraph maps $G^{*} \rightarrow H^{*}$ then $L f$ and $L g$ are homotopic digraph maps $L G^{*} \rightarrow$ $L H^{*}$. If $G^{*} \simeq H^{*}$ then also $L G^{*} \simeq L H^{*}$.

Proof. In the first statement, it suffices to consider the case of one-step homotopy between $f$ and $g$, which by (3.2) amounts to either $f(x) \equiv g(x)$ for all $x \in V_{G}$ or $g(x) \rightrightarrows f(x)$ for all $x \in V_{G}$. Assume without loss of generality that

$$
f(x) \rightrightarrows g(x) \text { for all } x \in V_{G} \text {. }
$$

Then, for any loop $\psi \in L G^{*}, \psi: I_{n}^{*} \rightarrow G^{*}$, we have also

$$
f(\psi(i)) \rightrightarrows g(\psi(i)) \text { for all } i=0, \ldots, n,
$$

which implies that $f \circ \psi$ and $g \circ \psi$ are one-step homotopic and, hence, onestep $C$-homotopic. Therefore, the loops $f \circ \psi$ and $g \circ \psi$ as elements of $L H^{*}$ are either identical or connected by an edge in $L H^{*}$, that is

$$
(L f)(\psi) \rightrightarrows(L g)(\psi) \text { for all } \psi \in V_{L G} \text {. }
$$

Hence, $L f \simeq L g$, which finishes the proof of the first statement.

Since $L$ is a functor we obtain the proof of the rest part of the Theorem.

Corollary 4.26. For $n \geq 0$, the functor $\pi_{n}$ is well defined on the homotopy category of based digraphs.

Remark 4.27. The definition of higher homotopy groups $\pi_{n}\left(G^{*}\right)$ depends crucially on how we define edges in the loop-digraph $L G^{*}$. Our present definition uses for that one-step $C$-homotopy. There may be other definitions of edges in $L G^{*}$, for example, one could use for that the transformations of Theorem 4.13. By switching to the latter (or any other reasonable) definition of $L G^{*}$, the set of connected components of $L G^{*}$ remains unchanged, so that $\pi_{1}\left(G^{*}\right)=\pi_{0}\left(L G^{*}\right)$ is unchanged, but $\pi_{1}\left(L G^{*}\right)$ and, hence, $\pi_{2}\left(G^{*}\right)$ may become different. At present it is not quite clear what is the most natural choice of edges in $L G^{*}$. We plan to return to this question in the future research. 


\section{Application to graph coloring}

An an illustration of the theory of digraph homotopy, we give here a new proof of the classical lemma of Sperner, using the notion the fundamental group and $C$-homotopy.

Consider a triangle $A B C$ on the plane $\mathbb{R}^{2}$ and its triangulation $T$. The set of vertices of $T$ is colored with three colors $1,2,3$ in such a way that

- the vertices $A, B, C$ are colored with $1,2,3$ respectively;

- each vertex on any side of $A B C$ is colored with one of the two colors of the endpoints of the side (see Fig. 15).

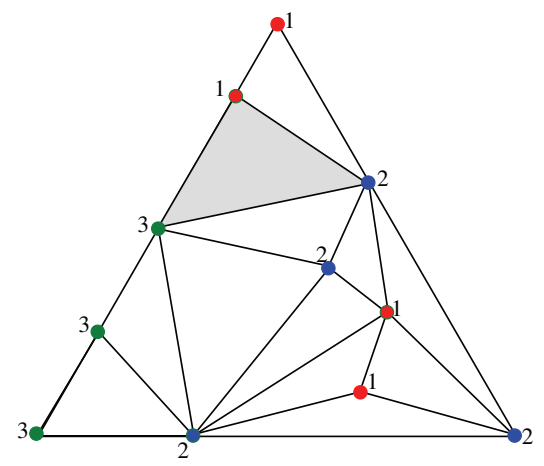

Figure 15: A Sperner coloring

The classical lemma of Sperner says that then there exists in $T$ a $3-$ color triangle, that is, a triangle, whose vertices are colored with the three different colors.

To prove this, let us first modify the triangulation $T$ so that there are no vertices on the sides $A B, A C, B C$ except for $A, B, C$. Indeed, if $X$ is a vertex on $A B$ then we move $X$ a bit inside the triangle $A B C$. This gives rise to a new triangle in the triangulation $T$ that is formed by $X$ and its former neighbors, say $Y$ and $Z$, on the edge $A B$ (while keeping all other triangles). However, since all $X, Y, Z$ are colored with two colors, no 3-color triangle emerges after that move. By induction, we remove all the vertices from the sides of $A B C$.

The triangulation $T$ can be regarded as a graph. Let us make it into a digraph $G$ by choosing the direction on the edges as follows. If the vertices 
$a, b$ are connected by an edge in $T$ then choose direction between $a, b$ using the colors of $a, b$ and the following rule:

$$
\begin{array}{ll}
1 \rightarrow 2, & 2 \rightarrow 3, \quad 3 \rightarrow 1 \\
1 \leftrightarrows 1, & 2 \leftrightarrows 2, \quad 3 \leftrightarrows 3
\end{array}
$$

Assume now that there is no 3 -color triangle in $T$. Then each triangle from $T$ looks in $G$ like

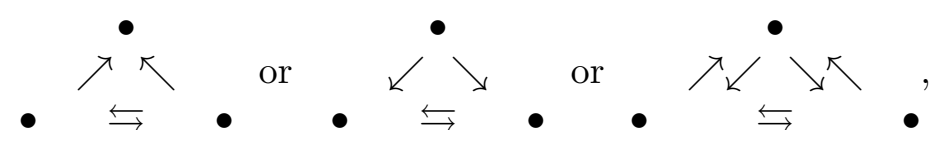

in particular, each of them contains a triangle in the sense of Theorem 4.13. Using the transformations (ii) and (iv) of Theorem 4.13 and the partition of $G$ into the triangles, we contract any loop on $G$ to an empty word (cf. Fig. 14), whence $\pi_{1}\left(G^{*}\right)=\{0\}$.

Consider now a colored cycle $S_{3}$

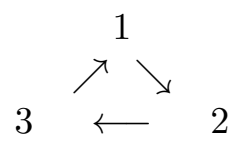

and the following two maps: $f: G \rightarrow S_{3}$ that preserves the colors of the vertices and $g: S_{3} \rightarrow G$ that maps the vertices $1,2,3$ of $S_{3}$ onto $A, B, C$, respectively. Both $f, g$ are digraph maps, which for the case of $f$ follows from the choice (5.1) of directions of the edges of $G$. Since $f \circ g=\mathrm{id}_{S_{3}}$, we obtain that $\pi_{1}(f \circ g)=\pi_{1}(f) \circ \pi_{1}(g)$ is an isomorphism of $\pi_{1}\left(S_{3}\right) \simeq \mathbb{Z}$ onto itself, which is not possible by $\pi_{1}\left(G^{*}\right)=\{0\}$.

\section{Homology and homotopy of (undirected) graphs}

A homotopy theory of undirected graphs was constructed in [1] and [2] (see also [4]). Here we show that this theory can be obtained from our homotopy theory of digraphs as restriction to a full subcategory. The same restriction enables us to define a homotopy invariant homology theory of undirected graphs such that the classical relation between fundamental group and the first homology group given by Theorem 4.23 is preserved. In particular, the so obtained homology theory for graphs answers a question raised in $[1$, p.32].

To distinguish digraphs (see Definition 2.1) and (undirected) graphs (see Definition 3.1 below) we use the following notations. To denote a digraph 
and its sets of vertices and edges, we use as in the previous sections the standard font as $G=\left(V_{G}, E_{G}\right)$. To denote a graph and its sets of vertices and edges, we will use a bold font, for example, $\mathbf{G}=\left(\mathbf{V}_{\mathbf{G}}, \mathbf{E}_{\mathbf{G}}\right)$. The bold font will also be used to denoted the maps between graphs.

Definition 6.1. (i) A graph $\mathbf{G}=\left(\mathbf{V}_{\mathbf{G}}, \mathbf{E}_{\mathbf{G}}\right)$ is a couple of a set $\mathbf{V}_{\mathbf{G}}$ of vertices and a subset $\mathbf{E}_{\mathbf{G}} \subset\left\{\mathbf{V}_{\mathbf{G}} \times \mathbf{V}_{\mathbf{G}} \backslash \operatorname{diag}\right\}$ of non-ordered pairs of vertices that are called edges. Any edge $(v, w) \in \mathbf{E}_{\mathbf{G}}$ will be also denoted by $v \sim w$.

(ii) A morphism from a graph $\mathbf{G}=\left(\mathbf{V}_{\mathbf{G}}, \mathbf{E}_{\mathbf{G}}\right)$ to a graph $\mathbf{H}=\left(\mathbf{V}_{\mathbf{H}}, \mathbf{E}_{\mathbf{H}}\right)$ is a map

$$
\mathbf{f}: \mathbf{V}_{\mathbf{G}} \rightarrow \mathbf{V}_{\mathbf{H}}
$$

such that for any edge $v \sim w$ on $\mathbf{G}$ we have either $\mathbf{f}(v)=\mathbf{f}(w)$ or $\mathbf{f}(v) \sim$ $\mathbf{f}(w)$. We will refer to morphisms of graphs as graph maps.

To each graph $\mathbf{G}=\left(\mathbf{V}_{\mathbf{G}}, \mathbf{E}_{\mathbf{G}}\right)$ we associate a digraph $G=\left(V_{G}, E_{G}\right)$ where $V_{G}=\mathbf{V}_{\mathbf{G}}$ and $E_{G}$ is defined by the condition $v \rightarrow w \Leftrightarrow v \sim w$. Clearly, the $\operatorname{digraph} G$ satisfies the condition $w \rightarrow v \Leftrightarrow v \rightarrow w$. Any digraph with this property will be called a double digraph.

The set of all graphs with graph maps forms a category (which was also introduced by [1] and [2]), that will be denoted by $\mathcal{G}$.

The assignment $\mathbf{G} \mapsto G$ and a similar assignment $\mathbf{f} \mapsto f$ of maps, that is well defined, provide a functor $\mathcal{O}$ from $\mathcal{G}$ to $\mathcal{D}$. It is clear that the image $\mathcal{O}$ is a full subcategory $\mathcal{O}(\mathcal{G})$ of $\mathcal{D}$ that consists of double digraphs, such that the inverse functor $\mathcal{O}^{-1}: \mathcal{O}(\mathcal{G}) \rightarrow \mathcal{G}$ is well defined.

Definition 6.2. For two graphs $\mathbf{G}=\left(\mathbf{V}_{\mathbf{G}}, \mathbf{E}_{\mathbf{G}}\right)$ and $\mathbf{H}=\left(\mathbf{V}_{\mathbf{H}}, \mathbf{E}_{\mathbf{H}}\right)$ define the Cartesian product $\mathbf{G} \bullet \mathbf{H}$ as a graph with the set of vertices $\mathbf{V}_{\mathbf{G}} \times \mathbf{V}_{\mathbf{H}}$ and with the set of edges as follows: for $x, x^{\prime} \in \mathbf{V}_{\mathbf{G}}$ and $y, y^{\prime} \in \mathbf{V}_{\mathbf{H}}$, we have $(x, y) \sim\left(x^{\prime}, y^{\prime}\right)$ in $\mathbf{G} \bullet \mathbf{H}$ if and only if

$$
\text { either } x^{\prime}=x \text { and } y \sim y^{\prime} \text {, or } x \sim x^{\prime} \text { and } y=y^{\prime} \text {. }
$$

The comparison of Definitions 2.3 and 6.2 yields the following statement.

Lemma 6.3. The functors $\mathcal{O}$ and $\mathcal{O}^{-1}$ preserve the product $\square$, that is

$$
\mathcal{O}(\mathbf{G} \boxminus \mathbf{H})=G \boxminus H, \quad \mathcal{O}^{-1}(G \boxminus H)=\mathbf{G} \boxminus \mathbf{H} .
$$

By definition, a line graph is a graph $\mathbf{J}_{n}=(\mathbf{V}, \mathbf{E})$ with $\mathbf{V}=\{0,1, \ldots, n\}$ and $\mathbf{E}=\{k \sim k+1 \mid 0 \leq k \leq n-1\}$. Let $\mathbf{J}=\{0 \sim 1\}$ be the line graph with two vertices. Let $J_{n}=\mathcal{O}\left(\mathbf{J}_{n}\right)$ and $J=\mathcal{O}(\mathbf{J})$. 
Definition 6.4. [2] Let $\mathbf{G}, \mathbf{H}$ be two graphs.

(i) Two graph maps $\mathbf{f}, \mathbf{g}: \mathbf{G} \rightarrow \mathbf{H}$ are called homotopic if there exists a line graph $\mathbf{J}_{n}(n \geq 0)$ and a graph map $\mathbf{F}: \mathbf{G} \boxminus \mathbf{J}_{n} \rightarrow \mathbf{H}$ such that

$$
\left.\mathbf{F}\right|_{\mathbf{G} \square\{0\}}=\mathbf{f}_{0} \text { and }\left.\mathbf{F}\right|_{\mathbf{G} \square\{n\}}=\mathbf{f}_{1}
$$

In this case we shall write $\mathbf{f} \simeq \mathbf{g}$.

(ii) The graphs $\mathbf{G}$ and $\mathbf{H}$ are called homotopy equivalent if there exist graph maps $\mathbf{f}: \mathbf{G} \rightarrow \mathbf{H}$ and $\mathbf{g}: \mathbf{H} \rightarrow \mathbf{G}$ such that

$$
\mathbf{f} \circ \mathbf{g} \simeq \mathrm{id}_{\mathbf{H}}, \quad \mathbf{g} \circ \mathbf{f} \simeq \mathrm{id}_{\mathbf{G}} .
$$

In this case we shall write $\mathbf{H} \simeq \mathbf{G}$. The maps $\mathbf{f}$ and $\mathbf{g}$ are as in (6.1) called homotopy inverses of each other.

The relation " $\simeq$ " is an equivalence relation on the set of graph maps and on the set of graphs (see [2]).

Proposition 6.5. Let $\mathbf{f}, \mathbf{g}: \mathbf{G} \rightarrow \mathbf{H}$ be graph maps. The maps $\mathbf{f}$ and $\mathbf{g}$ are homotopic if and only if the digraph maps $f=\mathcal{O}(\mathbf{f})$ and $g=\mathcal{O}(\mathbf{g})$ are homotopic.

Proof. Let $\mathbf{F}: \mathbf{G} \odot \mathbf{J}_{n} \rightarrow \mathbf{H}$ be a homotopy between $\mathbf{f}$ and $\mathbf{g}$ as in Definition 6.4. The natural digraph inclusion $I_{n} \rightarrow J_{n}$ (where $I_{n} \in \mathcal{I}$ is arbitrary) induces the digraph inclusion $\Theta: G \boxminus I_{n} \rightarrow G \boxminus J_{n}$. Applying functor $\mathcal{O}$ and Lemma 6.3 we obtain a digraph map $F:=G \boxminus J_{n} \rightarrow H$ such that the composition $F \circ \Theta: G \sqcup I_{n} \rightarrow H$ provides a digraph homotopy. Now let $F: G \unrhd I_{n} \rightarrow H$ be a digraph homotopy as in Definition 3.1 between two double digraphs. Define a digraph map $F^{\prime}: G \boxminus J_{n} \rightarrow H$ on the set of vertices by $F^{\prime}(x, i)=F(x, i)$. Since $H$ is a double digraph, this definition is correct. Applying functor $\mathcal{O}^{-1}$ and Lemma 6.3 we obtain a graph homotopy $\mathbf{F}^{\prime}: \mathbf{G} \odot \mathbf{J}_{n} \rightarrow \mathbf{H}$.

Denote by $\mathcal{D}^{\prime}$ the homotopy category of digraphs. The objects of this category are digraphs, and the maps are classes of homotopic digraphs maps. Similarly, denote by $\mathcal{G}^{\prime}$ the homotopy category of graphs and by $\mathcal{O}\left(\mathcal{G}^{\prime}\right)$ the homotopy category of double digraphs.

Proposition 6.5 implies the following.

Corollary 6.6. The functors $\mathcal{O}$ and $\mathcal{O}^{-1}$ induce an equivalence between homotopy category of graphs and homotopy category of double digraphs 
Definition 6.7. Let $\mathbb{K}$ be a commutative ring with unity. Define homology groups of a graph $\mathbf{G}$ with coefficients in $\mathbb{K}$ as follows: $H_{n}(\mathbf{G}, \mathbb{K})$ : = $H_{n}(G, \mathbb{K})$ where $G=\mathcal{O}(\mathbf{G})$.

The following statement follows from Theorem 3.3 and Proposition 6.5.

Proposition 6.8. The homology groups of a graph $\mathbf{G}$ with coefficients $\mathbb{K}$ are homotopy invariant.

A (induced) subgraph $\mathbf{H}$ of a graph $\mathbf{G}$ is a graph whose set of vertices is a subset of that of $\mathbf{G}$ and the edges of $\mathbf{H}$ are all those edges of $\mathbf{G}$ whose adjacent vertices belong to $\mathbf{H}$.

Definition 6.9. Let $\mathbf{G}$ be a graph and $\mathbf{H}$ be its subgraph. $\operatorname{id}_{\mathbf{H}}$.

(i) A retraction of $\mathbf{G}$ onto $\mathbf{H}$ is a graph map $\mathbf{r}: \mathbf{G} \rightarrow \mathbf{H}$ such that $\left.\mathbf{r}\right|_{\mathbf{H}}=$

(ii) A retraction $\mathbf{r}: \mathbf{G} \rightarrow \mathbf{H}$ is called a deformation retraction if $\mathbf{i} \circ \mathbf{r} \simeq$ $\operatorname{id}_{\mathbf{G}}$, where $\mathbf{i}: \mathbf{H} \rightarrow \mathbf{G}$ is the natural inclusion map.

Note that the condition $\mathbf{i} \circ \mathbf{r} \simeq \mathrm{id}_{\mathbf{G}}$ is equivalent to the existence of a graph morphism $\mathbf{F}: \mathbf{G} \bullet \mathbf{J}_{n} \rightarrow \mathbf{G}$ such that

$$
\left.\mathbf{F}\right|_{\mathbf{G} \triangleright\{0\}}=\operatorname{id}_{\mathbf{G}},\left.\quad \mathbf{F}\right|_{\mathbf{G} \sqcup\{n\}}=\mathbf{i} \circ \mathbf{r} .
$$

Similarly Proposition 3.5, a deformation retraction provides homotopy equivalence $\mathbf{G} \simeq \mathbf{H}$ with homotopy inverse maps $\mathbf{i}, \mathbf{r}$ (compare with [2, p.119]).

Example 6.10. ( $i)$ Let us define a cycle graph $\mathbf{S}_{n}(n \geq 3)$ as the graph that is obtained from $\mathbf{J}_{n}$ by identifying of the vertices $n$ and 0 . Then

$$
H_{p}\left(\mathbf{S}_{n}, \mathbb{K}\right)= \begin{cases}\mathbb{K}, & \forall n \text { and } p=0 \\ \mathbb{K}, & n \geq 5 \text { and } p=1 \\ 0, & \text { in other cases. }\end{cases}
$$

(ii) Let $\mathbf{G}$ be a star-like graph, that there is a vertex $a \in \mathbf{V}_{\mathbf{G}}$ such that $a \sim v$ for any $v \in \mathbf{V}_{\mathbf{G}}$. Then the map $\mathbf{r}: \mathbf{G} \rightarrow\{a\}$ is a deformation retraction which implies $\mathbf{G} \simeq\{a\}$ (cf. Example 3.11). Consequently, $H_{0}(\mathbf{G}, \mathbb{K})=\mathbb{K}$ and $H_{p}(\mathbf{G}, \mathbb{K})=0$ for all $p>0$.

(iii) If a graph $\mathbf{G}$ is a tree, then $\mathbf{G}$ is contractible (cf. Example 3.10). In particular, $H_{0}(\mathbf{G}, \mathbb{K})=\mathbb{K}$ and $H_{p}(\mathbf{G}, \mathbb{K})=0$ for all $p>0$. 
Definition 6.11. Let $\mathbf{f}: \mathbf{G} \rightarrow \mathbf{H}$ be a graph map. The cylinder $\mathrm{C}_{\mathbf{f}}$ of $\mathbf{f}$ is a graph with the set of vertices $\mathbf{V}_{\mathrm{C}_{\mathbf{f}}}=\mathbf{V}_{\mathbf{G}} \sqcup \mathbf{V}_{\mathbf{H}}$ and with the set of edges $\mathbf{E}_{\mathrm{C}_{\mathbf{f}}}$ that consists of all the edges from $\mathbf{E}_{\mathbf{G}}$ and $\mathbf{E}_{\mathbf{H}}$ as well as of the edges of the form $x \sim f(x)$ for all $x \in \mathbf{V}_{\mathbf{G}}$.

Analogously to Proposition 3.18, we obtain the following.

Proposition 6.12. We have a homotopy equivalence $\mathrm{C}_{\mathbf{f}} \simeq \mathbf{H}$.

Below we consider based graphs $\mathbf{G}^{*}$, where $*$ is a based vertex of $\mathbf{G}$. The based vertex of $\mathbf{J}_{n}$ will be usually 0 .

Definition 6.13. Let $\mathbf{G}$ be a graph. A path-map in a graph $\mathbf{G}$ is any graph map $\Phi: \mathbf{J}_{n} \rightarrow \mathbf{G}$. A based path on based graph $\mathbf{G}^{*}$ is a based map $\Phi: \mathbf{J}_{\mathbf{n}}^{*} \rightarrow \mathbf{G}^{*}$. A loop in $\mathbf{G}$ is a based path-map $\Phi: \mathbf{J}_{\mathbf{n}}^{*} \rightarrow \mathbf{G}^{*}$ such that $\Phi(n)=*$.

The inverse path-map and the concatenation of path-maps are defined similarly to Definition 4.16.

Definition 6.14. (i) A graph map $\mathbf{h}: \mathbf{J}_{n} \rightarrow \mathbf{J}_{m}$ is called shrinking if $\mathbf{h}(0)=$ $0, \mathbf{h}(n)=m$, and $\mathbf{h}(i) \leq \mathbf{h}(j)$ whenever $i \leq j$.

An extension of a based path-map $\Phi: \mathbf{J}_{m}^{*} \rightarrow \mathbf{G}^{*}$ is any path-map $\Phi^{E}=$ $\Phi \circ \mathbf{h}$ where $\mathbf{h}: \mathbf{J}_{n}^{*} \rightarrow \mathbf{J}_{m}^{*}$ is shrinking. An extension $\Phi^{E}$ is called a stabilization of $\Phi$ if the shrinking map $\mathbf{h}$ satisfies the condition $\left.\mathbf{h}\right|_{\mathbf{J}_{m}}=$ id. A stabilization of $\Phi$ will be denoted by $\Phi^{S}$.

(ii) Two loops $\Phi, \Psi$ in a based graph $\mathbf{G}^{*}$ are called $S$-homotopic if there exist stabilizations $\Phi^{S}, \Psi^{S}$ which are homotopic. In this case we shall write $\Phi \stackrel{S}{\simeq} \Psi$. This is an equivalence relation and equivalence class of a loop $\Phi$ will be denoted by $[\Phi]$ (cf. [1] and [2]).

Define a set $\pi_{1}\left(\mathbf{G}^{*}\right)$ as the set of $S$-equivalence classes of loops in $\mathbf{G}^{*}$, and the product in $\pi_{1}\left(\mathbf{G}^{*}\right)$ by $[\Phi] \cdot[\Psi]:=[\Phi \vee \Psi]$. Let $\mathbf{e}: \mathbf{J}_{0}^{*} \rightarrow \mathbf{G}^{*}$ be the trivial loop.

Proposition 6.15. [1], [2, Proposition 5.6] The set $\pi_{1}\left(\mathbf{G}^{*}\right)$ with the product defined above and with the neutral element $[\mathbf{e}]$ is a group, that will be referred to as a fundamental group of the graph $\mathbf{G}^{*}$ and denoted by $\pi_{1}\left(\mathbf{G}^{*}\right)$. 
Definition 6.16. Consider two based path-maps

$$
\Phi: \mathbf{J}_{n}^{*} \rightarrow \mathbf{G}^{*} \text { and } \Psi: \mathbf{J}_{m}^{*} \rightarrow \mathbf{G}^{*}
$$

An one-step $C$-homotopy from $\Phi$ to $\Psi$ is given by a shrinking map $\mathbf{h}: \mathbf{J}_{n} \rightarrow$ $\mathbf{J}_{m}$ such that the map $\mathbf{F}: \mathbf{V}_{\mathrm{C}_{\mathbf{h}}} \rightarrow \mathbf{V}_{\mathbf{G}}$ given by

$$
\left.\mathbf{F}\right|_{\mathbf{J}_{n}}=\Phi \quad \text { and }\left.\quad \mathbf{F}\right|_{\mathbf{J}_{m}}=\Psi
$$

is a graph map from $\mathrm{Ch}_{\mathbf{h}}$ to $\mathbf{G}$.

The path-maps $\Phi$ and $\Psi$ are said to be $C$-homotopic if there exists a sequence of one-step $C$-homotopies that connect $\Phi$ and $\Psi$. We shall write in this case $\Phi \stackrel{C}{\simeq} \Psi$.

The following statement follows immediately from definitions of the functor $\mathcal{O}$ and cylinder of the graph and digraph maps.

Lemma 6.17. Let $\mathbf{h}: \mathbf{G} \rightarrow \mathbf{H}$ be a graph map. There exists a natural digraph inclusion $\mathrm{C}_{h} \rightarrow \mathcal{O}\left(\mathbf{C}_{\mathbf{h}}\right)$, where $\mathrm{C}_{h}$ is a cylinder of the digraph map $h: G \rightarrow H$.

Theorem 6.18. Let $G^{*}$ be a based double digraph. We have a natural isomorphism of fundamental groups $\pi_{1}\left(\mathbf{G}^{*}\right) \cong \pi_{1}\left(G^{*}\right)$ where $\mathbf{G}^{*}=\mathcal{O}^{-1}\left(G^{*}\right)$.

Proof. Let $\Phi: \mathbf{J}_{n}^{*} \rightarrow \mathbf{G}^{*}$ be a based loop. Denote by $I_{n}^{s}$ the special line digraph with the vertices $0,1, \ldots, n$ and edges $i \rightarrow i+1$ for all $i=0, \ldots, n-1$. There is a natural inclusion $\tau: I_{n}^{s} \rightarrow J_{n}$. The composition $\mathcal{O}(\Phi) \circ \tau: I_{n}^{s *} \rightarrow$ $G^{*}$ defines a based loop $\phi$ in $G^{*}$. At first we would like to prove, that the correspondence $\Phi \longrightarrow \mathcal{O}(\Phi) \circ \tau=\phi$ provides a well defined map of sets

$$
\mathcal{O}_{*}: \pi_{1}\left(\mathbf{G}^{*}\right) \rightarrow \pi_{1}\left(G^{*}\right), \quad \mathcal{O}_{*}([\Phi]) \mapsto[\mathcal{O}(\Phi) \circ \tau]=[\phi]
$$

Let $\Phi: \mathbf{J}_{k}^{*} \rightarrow \mathbf{G}^{*}, \Psi: \mathbf{J}_{m}^{*} \rightarrow \mathbf{G}^{*}$ be loops and $\Phi \stackrel{S}{\simeq} \Psi$. The homotopic stabilizations $\Phi^{S}$ and $\Psi^{S}$ provide one-step $C$-homotopies $\Phi^{S} \stackrel{C}{\simeq} \Phi, \Psi^{S} \stackrel{C}{\simeq} \Psi$. A homotopy between $\Phi^{S}$ and $\Psi^{S}$ provides $C$-homotopy $\Phi^{S} \stackrel{C}{\simeq} \Psi^{S}$. Since $C$ homotopy is an equivalence relation, we obtain $\Phi \stackrel{C}{\simeq} \Psi$. Now by Lemma 6.17 we obtain that $\phi \stackrel{C}{\simeq} \psi$. That is the map $\mathcal{O}_{*}$ is well defined, and it is easy to see that this is a homomorphism of groups. This is an epimorphism as follows from Proposition 4.12. 
Digraph maps

$$
\phi: I_{n}^{s *} \rightarrow G^{*}, \quad \psi: I_{m}^{s *} \rightarrow G^{*}
$$

define graphs maps

$$
\Phi: \mathbf{J}_{n} \rightarrow \mathbf{G}^{*}, \quad \Phi: \mathbf{J}_{m} \rightarrow \mathbf{G}^{*}
$$

such that $\mathcal{O}(\Phi) \circ \tau=\phi$ and $\mathcal{O}(\Psi) \circ \tau=\psi$. A one-step $C$-homotopy $\phi \stackrel{C}{\simeq} \psi$ implies a one-step $C$-homotopy $\Phi \stackrel{C}{\simeq} \Psi$. That implies that $\Phi^{E}$ is homotopic to $\Psi$ or vice versa. To finish the proof of the Theorem, it suffices to prove that $\Phi \stackrel{S}{\simeq} \Psi$. But this follows directly from definition of fundamental group of graph in [1] and [2].

Theorem 6.19. For any based graph $\mathbf{G}^{*}$ we have an isomorphism

$$
\pi_{1}\left(\mathbf{G}^{*}\right) /\left[\pi_{1}\left(\mathbf{G}^{*}\right), \pi_{1}\left(\mathbf{G}^{*}\right)\right] \cong H_{1}(\mathbf{G}, \mathbb{Z})
$$

where $\left[\pi_{1}\left(\mathbf{G}^{*}\right), \pi_{1}\left(\mathbf{G}^{*}\right)\right]$ is a commutator subgroup.

Proof. Follows from Theorems 6.18 and 4.23 .

Definition 6.20. [1, p.41] Let $\mathbf{G}^{*}$ be a based graph.

(i) A based path graph $\mathbf{P G}^{*}$ is a graph with the set of vertices $\mathbf{V}_{\mathbf{P G}^{*}}=$ $\left\{\Phi: \mathbf{J}_{n} \rightarrow \mathbf{G}^{*}\right\}$, a base vertex $*: \mathbf{J}_{0} \rightarrow \mathbf{G}^{*}$, and there is an edge $\Phi \sim \Psi$ if and only $\Phi^{S} \simeq \Psi$ or $\Phi \simeq \Psi^{S}$.

(ii) A based loop graph $\mathbf{L} \mathbf{G}^{*}$ is a based sub-graph of $\mathbf{P} \mathbf{G}^{*}$ with the set of vertices $\mathbf{V}_{\mathbf{L G}} \mathbf{G}^{*}=\left\{\Phi: \mathbf{J}_{n} \rightarrow \mathbf{G}^{*} \mid \Phi(n)=*\right\}$ and with the restricted from $\mathbf{P} \mathbf{G}^{*}$ set of vertices.

(iii) Define higher homotopy groups $\pi_{n}\left(\mathbf{G}^{*}\right):=\pi_{n-1}\left(\mathbf{L G}^{*}\right)$ for $n \geq 2$.

Proposition 6.21. Let $G^{*}=\mathcal{O}\left(\mathbf{G}^{*}\right)$ be a based double digraph. Then $L G^{*}$ be a double digraph and we have a natural inclusion $\mathbf{1}: \mathbf{L G}^{*} \subseteq \mathcal{O}^{-1}\left(L G^{*}\right)$ that is an identity map on the set of vertices. This map induces a homomorphism of homotopy groups $\pi_{n}\left(\mathbf{L G}^{*}\right) \rightarrow \pi_{n}\left(L G^{*}\right)$ for $n \geq 1$ and an isomorphism for $n=0$.

Proof. The proof that $L G^{*}$ is a double digraph is similar to the proof of Proposition 6.5 and the graph map 1 is well defined by Lemma 6.17. Then the result follows. 


\section{References}

1. Eric Babson, Helene Barcelo, Mark de Longueville, and Reinhard Laubenbacher, Homotopy theory of graphs, Journal Algebr. Comb. 24 (2006), 31-44.

2. Helene Barcelo, Xenia Kramer, Reinhard Laubenbacher, and Christopher Weaver, Foundations of a connectivity theory for simplicial complexes, Advances in Appl. Mathematics 26 (2001), 97-128.

3. Marshall M. Cohen, A course in simple-homotopy theory, Berlin, New York: Springer-Verlag, 1973.

4. Anton Dochtermann, Homotopy groups of Hom complexes of graphs, Journal of Combinatorial Theory, Series A 116 (2009), 18-194.

5. Alexander Grigor'yan, Yong Lin, Yuri Muranov, and Shing-Tung Yau, Homologies of path complexes and digraphs, Math arXiv: $1207.2834 \mathrm{v} 4$ (2013).

6. Cohomology of digraphs and (undirected) graphs, to appear in Asian Journal of Mathematics (2014).

7. Alexander Grigor'yan, Yuri Muranov, and Shing-Tung Yau, Graphs associated with simplicial complexes, Homology, Homotopy, and Applications 16 (2014), 295-311.

8. Allen Hatcher, Algebraic Topology, Cambridge University Press, 2002.

9. Alexander V. Ivashchenko, Contractible transformations do not change the homology groups of graphs, Discrete Math. 126 (1994), 159-170.

10. S. MacLane, Homology, Die Grundlehren der mathematischen Wissenschaften. Bd. 114. Berlin-Göttingen-Heidelberg: Springer-Verlag, 522 pp., 1963.

11. P. Ribenboim, Algebraic structures on graphs, Algebra Universalis 16 (1983), 105-123.

12. Edwin H. Spanier, Algebraic topology, Berlin: Springer-Verlag, 528 p., 1995.

13. Mohamed Elamine Talbi and Djilali Benayat, Homology theory of graphs, Mediterranean J. of Math 11 (2014), 813-828.

Alexander Grigor'yan

Department of Mathematics, University of Bielefeld 
33501 Bielefeld, Germany

E-mail: grigor@math.uni-bielefeld.de

Yong Lin

Department of Mathematics, Renmin University of China

59 Zhongguancun Street, Haidian,

Beijing, China, 100872

E-mail: linyong01@ruc.edu.cn

Yuri Muranov

Department of Mathematics, University of Warmia and Mazury

ul. Sloneczna 54,

10-710 Olsztyn, Poland

E-mail:muranov@matman.uwm.edu.pl

Shing-Tung Yau

Department of Mathematics, Harvard University

Cambridge MA 02138, USA

E-mail: yau@math.harvard.edu 